\title{
Multidimensional Lagrange Problems of Optimization in a Fixed Domain and an Application to a Problem of Magnetohydrodynamics

\author{
LAMBERTO CESARI
}

In previous papers [3-5] we have given existence theorems for solutions in Sobolev spaces of multidimensional Lagrange problems of optimization in a fixed domain, bounded or unbounded. In the present paper we shall apply the previous results to the case where the partial differential equations are written in canonic form.

The canonic form which we take into consideration is the one proposed by RASHEVSKY in his book [9, pp. 323-324]. THOMAS \& TrTT [10] consider essentially the same form though this form is not written explicitly. RASHEvSKY has pointed out the generality of the proposed form. In [6-8] LURIE discusses necessary conditions for Lagrange problems of optimization with partial differential equations in canonic form. In particular, in [7, 8] LURIE discusses necessary conditions for an optimal solution in a two dimensional problem of magnetohydrodynamic channel flow, though with no previous existence analysis.

In the present paper we shall show that our existence theorems yield the existence of an optimal weak solution for LURIE's problem of magnetohydrodynamics. This optimal solution, of which we prove the existence, is weaker, however, than those considered by LURIE, namely it is a weak solution in the sense of Gamkrelidze with state functions belonging to a Sobolev space $W_{2}^{1}$.

\section{Part I.}

\section{§ 1. Partial Differential Equations in Canonic Form}

To simplify our exposition we shall assume that the number of independent variables is 2 , and we shall denote them by $x, y$. Let $G$ denote an open subset of the $x y$-plane $E_{2}$, and denote by $z(x, y)=\left(z^{1}, \ldots, z^{n}\right), u(x, y)=\left(u^{1}, \ldots, u^{m}\right)$, real functions of $x, y$ in $G$. We shall designate $z^{i}, i=1, \ldots, n$, as the state variables, and $u^{j}, j=1, \ldots, m$, as the control variables, all of which are in any case dependent variables.

We shall consider (with RASHEVSKY \& LURIE) partial differential systems of the form

$$
\begin{gathered}
\partial z^{i} / \partial x=X_{i}(x, y, z, u), \quad \partial z^{i} / \partial y=Y_{i}(x, y, z, u), \quad i=1,2, \ldots, n, \\
\partial X_{i} / \partial y=\partial Y_{i} / \partial x, \quad i=1,2, \ldots, n,
\end{gathered}
$$

6 Arch. Rational Mech. Anal., Vol. 29 
where in the latter equation the derivatives are taken with respect to all arguments of $X_{i}$ and $Y_{i}$. (We denote here as variables $u^{j}, j=1, \ldots, m$, both classes of RASHEVSKY's \& LURIE's variables $\zeta^{r}$ and $u^{s}$. See [8] and [9].)

Equations $(1,2)$ presuppose an amount of differentiability properties which will not be realized in the statements of our existence theorems below. Thus, we shall replace (2) by the weaker form

$$
\iint_{G}\left(X_{i} \varphi_{y}-Y_{i} \varphi_{x}\right) d x d y=0, \quad i=1, \ldots, n,
$$

for every scalar function $\varphi(x, y),(x, y) \in G$, of class $C^{\infty}$ in $G$ and having compact support contained in $G$ (briefly of class $C_{0}^{\infty}$ in $G$ ), and in view of (1), equation (3) can be replaced by

$$
\iint_{G}\left(z_{x}^{i} \varphi_{y}-z_{y}^{i} \varphi_{x}\right) d x d y=0
$$

for every $\varphi \in C_{0}^{\infty}$. Whenever the functions $X_{i}, Y_{i}, z^{i}, u^{j}$ are continuously differentiable with respect to all their arguments, then relations (4), or (3), yield equations (2).

As stated by RASHEVSKY (loc. cit.) equations (1) present a standard form of a remarkably general class of systems of partial differential equations, which all can be written in the form (1) (with the number of dependent variables increased if necessary). The presence of an increased number of variables is typical in the majority of applications. For instance, the Helmholtz equation $z_{x x}^{1}+z_{y y}^{1}+u z^{1}=0$ can be written in the form (1) with $n=3, m=3$ as follows (with $u$ replaced by $u, v^{1}, v^{2}$, and $z^{1}$ by $\left.z^{1}, z^{2}, z^{3}\right)$ :

$$
z_{x}^{1}=z^{2}, \quad z_{y}^{1}=z^{3}, \quad z_{x}^{2}=-v^{2}-u z^{1}, \quad z_{y}^{2}=v^{1}, \quad z_{x}^{3}=v^{1}, \quad z_{y}^{3}=v^{2} .
$$

The wave equation $z_{y y}^{1}-\left(k z_{x}^{1}\right)_{x}=0$ can be written in the form above with $n=2$, $m=2$ :

$$
z_{x}^{1}=-v^{1} / k, \quad z_{y}^{1}=v^{2}, \quad z_{x}^{2}=v^{2}, \quad z_{y}^{2}=-v^{1} .
$$

The form (1) corresponds to problems in a weaker form than those given by the differential equation of higher order. For instance, the system with $n=2, m=3$,

$$
z_{x}^{1}=v^{1}, \quad z_{y}^{1}=-v^{2}+u, \quad z_{x}^{2}=v^{2}, \quad z_{y}^{2}=v^{1}
$$

yields the system $\Delta z^{1}=u_{y}, \Delta z^{2}=u_{x}$ (with $\Delta=\left(\partial^{2} / \partial x^{2}\right)+\left(\partial^{2} / \partial y^{2}\right)$ ) only if $u$ is differentiable. In other words, the solutions of systems (1) can be thought of as generalized solutions of some original higher order partial differential equations. We shall further generalize the concept of solution in a number of ways.

\section{§ 2. Notations for Lagrange Problems with Partial Differential Equations in Canonic Form}

We shall first introduce unilateral constraints in a form more general than those considered by LURIE, namely in a form similar to those of our previous papers $[3-5]$.

For every $(x, y) \in \operatorname{cl} G$ let $A(x, y)$ denote a given nonempty subset of the $z$-space $E_{n}$. We shall denote by $A$ the set of all $(x, y, z) \in E_{2} \times E_{n}$ with $(x, y) \in \operatorname{cl} G$, 
$z \in A(x, y)$. We shall assume below that $A$ is a compact, or at least closed, subset of $E_{2} \times E_{n}$. For every $(x, y, z) \in A$ let $U(x, y, z)$ be a nonempty subset of the $u$-space $E_{m}$, and let $M$ denote the set of all $(x, y, z, u)$ with $(x, y) \in \operatorname{cl} G, z \in A(x, y)$, $u \in U(x, y, z)$, or $(x, y, z) \in A, u \in U(x, y, z)$. We shall consider couples $z(x, y)=$ $\left(z^{1}, \ldots, z^{n}\right), u(x, y)=\left(u^{1}, \ldots, u^{m}\right),(x, y) \in G$, satisfying the constraints

$$
(x, y, z(x, y)) \in A, \quad u(x, y) \in U(x, y, z(x, y)) \quad \text { a.e. in } G .
$$

To simplify notations we shall often denote by $f$ the $2 n$-vector function $f(x, y, z, u)=\left(f_{1}, \ldots, f_{2 n}\right)$ defined on $M$ by

$$
f_{i}=X_{i}(x, y, z, u), \quad f_{n+i}=Y_{i}(x, y, z, u), \quad i=1, \ldots, n .
$$

We shall also denote, if needed, by $D z$ the $2 n$-vector $\left(z_{x}^{i}, i=1, \ldots, n, z_{y}^{i}, i=1, \ldots, n\right)$. Then the differential system (1) can now be written in the simple form

$$
D z=f(x, y, z, u) \text {. }
$$

This canonical system is a particular case of the systems of partial differential equations of any order we have considered in [3-5]. Most of the results below are particular cases of those of [3-5].

Given $\delta>0$ and a point $\left(x_{0}, y_{0}, z_{0}\right) \in A$, we shall denote by "closed neighborhood $N_{\delta}\left(x_{0}, y_{0}, z_{0}\right)$ of radius $\delta$ of $\left(x_{0}, y_{0}, z_{0}\right)$ in $A$ " the set of all $(x, y, z) \in A$ at a distance $\leqq \delta$ from $\left(x_{0}, y_{0}, z_{0}\right)$. Also, we shall denote by $U_{\varepsilon}$ the set of all points $u \in E_{m}$ at a distance $\leqq \varepsilon$ from a given set $U$. We shall say that $U(x, y, z)$ is metrically upper semicontinuous at the point $\left(x_{0}, y_{0}, z_{0}\right) \in A$ provided, given $\varepsilon>0$, there is some $\delta=\delta\left(x_{0}, y_{0}, z_{0}, \varepsilon\right)>0$ such that $U(x, y, z) \subset\left[U\left(x_{0}, y_{0}, z_{0}\right)\right]_{\varepsilon}$ for all $(x, y, z) \epsilon$ $N_{\delta}\left(x_{0}, y_{0}, z_{0}\right)$. We shall say that $U(x, y, z)$ is metrically upper semicontinuous in $A$ provided $U(x, y, z)$ has this property at every point $\left(x_{0}, y_{0}, z_{0}\right) \in A$. This concept of metric upper semicontinuity is most often used when the sets $U(x, y, z)$ are compact and all contained in a bounded part of $E_{m}$. In general, the sets $U(x, y, z)$ are only closed and not compact, and in these cases it has been found $[1-5]$ that analogous concepts of upper semicontinuity, more topological in character, are needed. We shall denote these properties as properties $(U)$ and $(Q)$.

First, given $\delta>0$ and a point $\left(x_{0}, y_{0}, z_{0}\right) \in A$ let us denote by $U\left(x_{0}, y_{0}, z_{0} ; \delta\right)$ the set

$$
U\left(x_{o}, y_{0}, z_{0} ; \delta\right)=\bigcup U(x, y, z),
$$

where $U$ ranges over all $(x, y, z) \in N_{\delta}\left(x_{0}, y_{0}, z_{0}\right)$. We shall say that $U(x, y, z)$ satisfies property $(U)$ at a point $\left(x_{0}, y_{0}, z_{0}\right) \in A$ provided

that is,

$$
U\left(x_{0}, y_{0}, z_{0}\right)=\bigcap_{\delta>0} \operatorname{cl} U\left(x_{0}, y_{0}, z_{0} ; \delta\right),
$$

$$
U\left(x_{0}, y_{0}, z_{0}\right)=\bigcap_{\delta} \mathrm{cl} \underset{(x, y, z) \in N_{\delta}\left(x_{0}, y_{0}, z_{0}\right)}{U} U(x, y, z) .
$$

We shall say that $U(x, y, z)$ satisfies property $(U)$ in $A$ if $U(x, y, z)$ satisfies property $(U)$ at every point $\left(x_{0}, y_{0}, z_{0}\right) \in A$. $A$ set $U(x, y, z)$ satisfying property $(U)$ is necessarily closed as the intersection of closed sets. Below we shall also consider 
the sets

$$
\begin{aligned}
Q(x, y, z) & =f(x, y, z, U(x, y, z)) \\
& =\left[\zeta=\left(\zeta^{1}, \ldots, \zeta^{2 n}\right) \mid \zeta=f(x, y, z, u), u \in U(x, y, z)\right] \subset E_{2 n},
\end{aligned}
$$

and other analogous ones which we shall introduce as needed. We shall say that such a set $Q(x, y, z)$ satisfies property $(Q)$ at a point $\left(x_{0}, y_{0}, z_{0}\right) \in A$ provided

that is,

$$
Q\left(x_{0}, y_{0}, z_{0}\right)=\bigcap_{\delta>0} \operatorname{cl} \operatorname{co} Q\left(x_{0}, y_{0}, z_{0} ; \delta\right),
$$

$$
Q\left(x_{0}, y_{0}, z_{0}\right)=\bigcap_{\delta} \operatorname{clco} \bigcup_{(x, y, z) \in N_{\delta}\left(x_{0}, y_{0}, z_{0}\right)} Q(x, y, z) .
$$

We shall say that $Q(x, y, z)$ satisfies property $(Q)$ in $A$ if $Q(x, y, z)$ satisfies property $(Q)$ at every point $\left(x_{0}, y_{0}, z_{0}\right) \in A$. $A$ set $Q(x, y, z)$ satisfying property $(Q)$ is necessarily closed and convex as the intersection of closed and convex subsets of $E_{2 n}$. Above, cl $E$ denotes the closure of a set $E$, and co $E$ denotes the convex hull of $E$. Thus, cl co $E$ denotes the closure of the convex hull of $E$.

As in the previous papers [3-5] and as usual we shall assume for $G$ and its boundary $S=\partial G$ a certain amount of regularity, and we shall say that $G$ is of class $K_{01}$. If $G$ is an open bounded set, by $G$ of class $K_{01}$ we shall mean that $K=\operatorname{cl} G=G \cup S$ is the union of finitely many nonoverlapping parts $K_{1}, \ldots, K_{J}$, each $K_{j}=T_{j}(R)$ being the $1-1$ image of a rectangle $R$ under a transformation $T_{j}$ which is continuous with its inverse $T^{-1}$, and both $T_{j}$ and $T_{j}^{-1}$ can be represented by means of functions of class $C^{1}, j=1, \ldots, J$. (Actually, it would be enough to assume that both $T_{j}$ and $T_{j}^{-1}$ are Lipschitzian of a given constant.) The further usual convention shall be made that the boundary $S$ of $G$ is the union of nonoverlapping arcs $\lambda_{s}$, each $\lambda_{s}$ being the image under $T_{j}$ of one side $l_{j}$ of $R$, or $\lambda_{s}=T_{j}\left(l_{j}\right)$ for only one $j$.

If $G$ is unbounded, we shall say that $G$ is of class $K_{01}$ if its closure $K=\mathrm{cl} G=$ $G \cup S$ is the countable union of nonoverlapping parts $K_{1}, K_{2}, \ldots$, each $K_{j}=T_{j}(R)$, $j=1,2, \ldots$, as before, and with the further assumptions that each set

$$
V_{N}=\bigcup_{j=1}^{N} K_{j}=\mathrm{cl} G_{N}
$$

is the closure of an open bounded set $G_{N}$ of class $K_{01}$, that every interval $R$ of $E_{v}$ has a nonempty intersection with at most finitely many $K_{j}$, and that $G=\bigcup G_{N}$, $G_{N} \subset G_{N+1}$, that is, $G$ is the union of the bounded open subsets $G_{N}$ all of class $K_{01}$.

Obviously, there are $\infty$-many decompositions as described above of sets $G$ of class $K_{01}$, bounded or unbounded. Any such decomposition will be called a typical representation of the set $G$ of class $K_{01}$. If $G$ is a bounded open set of class $K_{01}$ then, concerning the couples $z(x, y)=\left(z^{1}, \ldots, z^{n}\right), u(x, y)=\left(u^{1}, \ldots, u^{m}\right)$, we shall assume that each component $z^{i}$ belongs to a Sobolev space $W_{p_{i}}^{1}(G)$ with $p_{i} \geqq 1$, and that each component $u^{j}$ is measurable in $G$. As a consequence, each component $x^{i}$ has boundary values $\varphi^{i}$ on the boundary $S=\partial G$ of $G$ and $\varphi^{i}$ is of class $L_{p_{i}}$ on $S$. Also, each component $z^{i}$ has first order generalized partial derivatives $z_{x}^{i}=D_{x} z^{i}, z_{y}^{i}=D_{y} z^{i}, i=1, \ldots, n$, a.e. in $G$, and $z^{i}, z_{x}^{i}, z_{y}^{i} \in L_{p_{i}}(G)$. 
If $G$ is an unbounded open set of class $K_{01}$, and $G=\bigcup G_{N}, G_{N} \subset G_{N+1}$, one of its typical representations, then we shall assume again each component $u^{j}$ measurable in $G$, and each component $z^{i}$ restricted to $G_{N}$ belonging to a Sobolev space $W_{p_{l}}^{1}\left(G_{N}\right)$ for all $N$ (and the same $\left.p_{i} \geqq 1\right)$; briefly, $z^{i} \in W_{p_{i}}^{1}\left(G_{N}\right)$ for every $N$. Then each component $z^{i}$ has boundary values $\varphi^{i}$ on $S$ which are locally of class $L_{p}$ on $S$, and each $z^{i}$ has generalized first order partial derivatives $z_{x}^{i}=D_{x} z^{i}, z_{y}^{i}=D_{y} z^{i}$ a.e. in $G$ with $z^{i}, D_{x} z^{i}, D_{y} z^{i} \in L_{p_{i}}\left(G_{N}\right)$ for every $N$.

We shall now require a set $(B)$ of boundary conditions involving the boundary values of the functions $z^{i}, i=1, \ldots, n$. On these boundary conditions $(B)$, we shall assume the following closure property for the case of $G$ a bounded open set of class $K_{01}$ and for given numbers $p_{i} \geqq 1, i=1, \ldots, n:\left(P_{1}\right)$ if $z(x, y)=\left(z^{1}, \ldots, z^{n}\right)$, $z_{k}(x, y)=\left(z_{k}^{1}, \ldots, z_{k}^{n}\right),(x, y) \in G, k=1,2, \ldots$, are vector functions whose components $z^{i}, z_{k}^{i}$ belong to the Sobolev class $W_{p_{i}}^{1}(G)$, if $z_{k}^{i} \rightarrow z^{i}$ as $k \rightarrow \infty$ strongly in $L_{p_{t}}(G)$, if $D_{x} z_{k}^{i} \rightarrow D_{x} z^{i}, D_{y} z_{k}^{i} \rightarrow D_{y} z^{i}$ as $k \rightarrow \infty$ weakly in $L_{p_{i}}(G)$, and the boundary values $\varphi_{k}^{i}$ of $z_{k}^{i}$ on $S$ satisfy boundary conditions $(B), i=1, \ldots, n$, then the boundary values $\varphi^{i}$ of $z^{i}$ on $S$ satisfy boundary conditions $(B), i=1, \ldots, n$.

For $G$ an unbounded open set of class $K_{01}$ in a given typical representation $G=\bigcup G_{N}, G_{N} \subset G_{N+1}$, as described before, we shall replace $\left(P_{1}\right)$ by the analogous condition, say still $\left(P_{1}\right)$, where we assume only $z^{i}, z_{k}^{i} \in W_{p_{i}}^{1}\left(G_{N}\right)$ for every $N$, we assume only that $z_{k}^{i} \rightarrow z^{i}$ as $k \rightarrow \infty$ strongly in $L_{p_{i}}\left(G_{N}\right)$ for every $N$, and we assume only that $D_{x} z_{k}^{i} \rightarrow D_{x} z^{i}, D_{y} z_{k}^{i} \rightarrow D_{y} z^{i}$ weakly in $L_{p_{i}}\left(G_{N}\right)$ for every $N$.

For instance, if the boundary conditions $(B)$ are defined by requiring that some of the boundary values coincide with preassigned continuous functions $\varphi^{i}$ on certain arcs of $S=\partial G$, then by force of SoBoLEv's imbedding theorems [9], as well as by direct argument, we know that property $\left(P_{1}\right)$ is valid.

Beside the $2 n$-vector $f(x, y, z, u)$, or $f=\left[X_{i}, i=1, \ldots, n, Y_{i}, i=1, \ldots, n\right]$, we shall consider a scalar function $f_{0}(x, y, z, u)$ also defined on $M$, and we shall denote by $\tilde{f}$ the $(2 n+1)$-vector function $\tilde{f}(x, y, z, u)=\left(f_{0}, f\right)=\left(f_{0}, X_{i}, Y_{i}, i=\right.$ $1, \ldots, n)$.

Let us mention that for a function $z \in W_{p}^{1}(G)$ we shall denote by $D_{p}(z, G)$ and $\tilde{D}_{p}(z, G)$ the nonnegative numbers

$$
\begin{aligned}
& D_{p}(z, G)=\left(\iint_{G}\left[\left(D_{x} z\right)^{2}+\left(D_{y} z\right)^{2}\right]^{p / 2} d x d y\right)^{1 / p}, \\
& \tilde{D}_{p}(z, G)=D_{p}(z, G)+\left(\iint_{G}|z|^{p} d x d y\right)^{1 / p} .
\end{aligned}
$$

A pair $z(x, y)=\left(z^{1}, \ldots, z^{n}\right), u(x, y)=\left(u^{1}, \ldots, u^{m}\right), \quad(x, y) \in G$, with $z^{i} \in W_{p_{i}}^{1}(G)$ [z $z^{i} \in W_{p_{1}}^{1}\left(G_{N}\right)$ for every $N$ if $G$ is unbounded], $u^{j}$ measurable in $G$, satisfying $(x, y, z(x, y)) \in A, u(x, y) \in U(x, y, z(x, y)), D_{x} z^{i}=X_{i}(x, y, z(x, y), u(x, y)), D_{y} z^{i}=$ $Y_{i}(x, y, z(x, y), u(x, y))$ a.e. in $G, i=1, \ldots, n$, satisfying the boundary conditions $(B)$, and such that $f_{0}(x, y, z(x, y), u(x, y)) \in L_{1}(G)$, is said to be admissible. A class $\Omega$ of admissible pairs is said to be complete if, for any sequence $z_{k}, u_{k}, k=1,2, \ldots$, of admissible pairs all in $\Omega$ and any other admissible pair $z, u$ such that $z_{k} \rightarrow z$ in the sense described under $\left(P_{1}\right)$ [according as $G$ is bounded or unbounded], the pair $z, u$ belongs to $\Omega$. The class of all admissible pairs is obviously complete.

The Lebesgue integral

$$
I[z, u]=\iint_{G} f_{0}(x, y, z(x, y), u(x, y)) d x d y,
$$


where $z, u$ is any admissible pair, is said to be the cost functional, or performance index. We shall seek the minimum of $J[z, u]$ in classes $\Omega$ of pairs $(z, u)$ with $z^{i} \in W_{p_{i}}^{1}(G), p_{i} \geqq 1$ [or $z^{i} \in W_{p_{i}}^{1}\left(G_{N}\right)$ for every $N$ ], and $u^{j}$ measurable, satisfying the constraints, the boundary conditions, the differential equations, and possibly a number of integral inequalities concerning the functions $z^{i}$ which we shall mention below.

Requirement (2) in the weak form (4) is always satisfied by any function $z^{i}$ of a Sobolev class $W_{p}^{1}(G), p \geqq 1$. Indeed, any such function $z^{i}$ is the limit of smooth functions $z_{k}^{i}$ (at least of class $C^{2}$ ), for which the relations

$$
\frac{\partial}{\partial x}\left(\frac{\partial z_{k}^{i}}{\partial y}\right)-\frac{\partial}{\partial y}\left(\frac{\partial z_{k}^{i}}{\partial x}\right)=0
$$

are satisfied everywhere, and $z_{k}^{i} \rightarrow z$ strongly in $L_{p}(G)$, and $D_{x} z_{k}^{i} \rightarrow D_{x} z^{i}, D_{y} z_{k}^{i} \rightarrow D_{y} z^{i}$ weakly in $L_{p}(G)$ as $k \rightarrow \infty$. The differential relation above then implies

$$
\iint_{G}\left[\left(D_{x} z_{k}^{i}\right) \varphi_{y}-\left(D_{y} z_{k}^{i}\right) \varphi_{x}\right] d x d y=0
$$

for all $\varphi \in C_{0}^{\infty}(G)$, and since $\varphi_{x} \in C_{0}^{\infty}(G), \varphi_{y} \in C_{0}^{\infty}(G)$, we can take the limit in (5) as $k \rightarrow \infty$, and obtain

$$
\iint_{G}\left[\left(D_{x} z^{i}\right) \varphi_{y}-\left(D_{y} z^{i}\right) \varphi_{x}\right] d x d y=0
$$

for all $\varphi \in C_{0}^{\infty}(G)$. If $G$ is unbounded, the same argument applies provided for every $\varphi \in C_{0}^{\infty}(G)$ we take a $G_{N}$ with $N$ sufficiently large.

All we have to do therefore is to prove the existence of optimal solutions in Sobolev spaces as mentioned above. In the process of the regularization problem it is often proved that the minimizing solutions are actually smooth.

\section{§ 3. Existence Theorems for Lagrange Problems in Sobolev Classes $W_{p}^{1}, p>1$}

Existence Theorem 1. Let $G$ be bounded, open, and of class $K_{01}$, let $A$ be compact, let $U(x, y, z)$ be nonempty and compact for every $(x, y, z) \in A$, and let $U(x, y, z)$ be metrically upper semicontinuous in $A$. Let $\tilde{f}(x, y, z, u)=\left(f_{0}, f\right)=\left(f_{0}, X_{i}, Y_{i}\right.$, $i=1, \ldots, n)$ be continuous on $M$, and let us assume that the set $\tilde{Q}(x, y, z)$ of all $\tilde{\zeta}=\left(\zeta^{0}, \zeta\right) \in E_{2 n+1}$ with $\zeta^{0} \geqq f_{0}(x, y, z, u), \zeta=f(x, y, z, u), u \in U(x, y, z)$, is a convex subset of $E_{2 n+1}$ for every $(x, y, z) \in A$. Let $(B)$ be a system of boundary conditions satisfying property $\left(P_{1}\right)$. Let $\Omega$ be a non-empty complete class of admissible pairs $z(x, y)=\left(z^{1}, \ldots, z^{n}\right), \quad u(x, y)=\left(u^{1}, \ldots, u^{m}\right), \quad(x, y) \in G, \quad$ with $\quad z^{i} \in W_{p_{i}}^{1}(G), \quad p_{i}>1$, $i=1, \ldots, n, u^{j}$ measurable, $j=1, \ldots, m$, and satisfying given inequalities

$$
\begin{array}{cl}
\iint_{G}\left|z^{i}\right|^{p_{i}} d x d y \leqq N_{i} & \text { for } i \in\{\beta\}, \\
\iint_{G}\left|D_{x} z^{i}\right|^{p} d x d y \leqq N_{i 1} & \text { for } i \in\{\beta\}_{x}, \quad \iint_{G}\left|D_{y} z^{i}\right|^{p_{i}} d x d y \leqq N_{i 2} \quad \text { for } i \in\{\beta\}_{y}
\end{array}
$$

for certain given constants $N_{i}, N_{i 1}, N_{i 2}$ and all $i$ of certain systems $\{\beta\},\{\beta\}_{x},\{\beta\}_{y}$ of indices $1,2, \ldots, n$ (which may be empty). Then the cost functional $I[z, u]$ possesses an absolute minimum in $\Omega$. 
Assume now that $G$ is unbounded, open and of class $K_{01}$, and that $G=\bigcup G_{N}$, $G_{N} \subset G_{N+1}$, is a typical representation of $G$. Assume that $A$ is closed, but that for every closed finite interval $R \subset E_{2}$ the subset of all $(x, y, z) \in A$ with $(x, y) \in R$ is compact. Let us assume that

$$
f_{0}(x, y, z, u) \geqq-\psi(x, y) \quad \text { for all }(x, y, z, u) \geqq M,
$$

where $\psi(x, y) \geqq 0$ is a given $L$-integrable function in $G$. If we know that for every pair $z, u$ of $\Omega$ we have

$$
z^{i} \in W_{p i}^{1}(G), \quad i=1, \ldots, n,
$$

and that there are constants $M_{i}^{\prime}$, such that

$$
\tilde{D}_{p_{i}}\left(z^{i}, G\right) \leqq M_{i}^{\prime}, \quad i=1, \ldots, n,
$$

for every admissible pair $z, u$ of the class $\Omega$, then Theorem 1 still holds as above, and for the minimizing admissible pairs $z, u$ of the class $\Omega$ of which we assert the existence (at least one) we know that (8) and (9) hold.

Again assume that $G$ is unbounded, open, and of class $K_{01}$, and that $G=\bigcup G_{N}$, $G_{N} \subset G_{N+1}$, is a typical representation of $G$ as before. Assume that $A$ is closed, but that for every closed finite interval $R \subset E_{2}$ the subset of all $(x, y, z) \in A$ with $(x, y) \in R$ is compact, and assume that (7) is satisfied as before. Theorem 1 then holds in a particularly weak form. Indeed assume that for every pair $z, u$ of the class $\Omega$ we have $z^{i} \in W_{p_{i}}^{1}\left(G_{N}\right)$ for every $N, p_{i}>1, i=1, \ldots, n$. Assume that relations (6) hold in the weak form

$$
\begin{aligned}
\iint_{G}\left|z^{i}\right|^{p_{i}} d x d y \leqq N_{i}(N) & \text { for } i \in\{\beta\}, \\
\iint_{G_{N}}\left|D_{x} z^{i}\right|^{p_{i}} d x d y \leqq N_{i 1}(N) & \text { for } i \in\{\beta\}_{x}, \\
\iint_{G_{N}}\left|D_{y} z^{i}\right|^{p_{i}} d x d y \leqq N_{i 2}(N) & \text { for } i \in\{\beta\}_{y}
\end{aligned}
$$

for every $N$ and for certain constants $N_{i}(N), N_{i 1}(N), N_{i 2}(N)$ which may depend on $N$. Under these weak assumptions Theorem 1 still holds, and for the minimizing admissible pairs $(z, u)$ of the class $\Omega$ of which we assert the existence (at least one) we know that

$$
\tilde{D}_{p_{i}}\left(z^{i}, G_{N}\right) \leqq M_{i N}^{\prime}, \quad i=1, \ldots, n,
$$

for every $N$ and constants $M_{i N}^{\prime}$ which may depend on $N$.

Existence Theorem 2. Let $G$ be bounded, open, and of class $K_{01}$, let $A$ be closed, let $U(x, y, z)$ be nonempty and closed for every $(x, y, z) \in A$, and assume that $U(x, y, z)$ satisfies property $(U)$ in $A$. Let $\tilde{f}(x, y, z, u)=\left(f_{0}, f\right)=\left(f_{0}, X_{i}, Y_{i}\right.$, $i=1, \ldots, n)$ be continuous on $M$, and let us assume that the set $\tilde{Q}(x, y, z)$ of all $\tilde{\zeta}=\left(\xi^{0}, \zeta\right) \in E_{2 n+1}$ with $\zeta^{0} \geqq f_{0}(x, y, z, u), \zeta=f(x, y, z, u), u \in U(x, y, z)$, is closed and convex for every $(x, y, z) \in A$, and satisfies property $(Q)$ in $A$. Let $(B)$ be a system of boundary conditions satisfying property $\left(P_{1}\right)$. Let $\Omega$ be a nonempty complete class of admissible pairs $z(x, y)=\left(z^{1}, \ldots, z^{n}\right), u(x, y)=\left(u^{1}, \ldots, u^{m}\right)$, $(x, y) \in G$, with $z^{i} \in W_{p_{i}}^{1}(G), p_{i}>1, i=1, \ldots, n, u^{j}$ measurable in $G, j=1, \ldots, m$, 
and satisfying given inequalities

$$
\begin{gathered}
\iint_{G}\left|f_{0}(x, y, z(x, y), u(x, y))\right|^{p_{0}} d x d y \leqq N_{0}, \\
\iint_{G}\left|z^{i}\right|^{p_{i}} d x d y \leqq N_{i} \quad \text { for } i \in\{\beta\}, \\
\iint_{G}\left|D_{x} z^{i}\right|^{p_{i}} d x d y \leqq N_{i 1} \quad \text { for } i \in\{\beta\}_{x}, \quad \iint_{G}\left|D_{y} z^{i}\right|^{p_{i}} d x d y \leqq N_{i 2} \quad \text { for } i \in\{\beta\}_{y}
\end{gathered}
$$

for certain given constants $p_{0}>1, N_{0}, N_{i}, N_{i 1}, N_{i 2}$, and all $i$ of certain systems $\{\beta\},\{\beta\}_{x},\{\beta\}_{y}$ of indices $1,2, \ldots, n$ (which may be empty). Assume that $(z, u) \in \Omega$, $I[z, u] \leqq L_{0}$ implies

$$
\begin{gathered}
\iint_{G}\left|z^{i}\right|^{p_{i}} d x d y \leqq L_{i}, \\
\iint_{G}\left|D_{x} z^{i}\right|^{p_{t}} d x d y \leqq L_{i 1}, \quad \iint_{G}\left|D_{y} z^{i}\right|^{p_{t}} d x d y \leqq L_{i 2},
\end{gathered}
$$

for all $i=1, \ldots, n$ which are not in $\{\beta\},\{\beta\}_{x},\{\beta\}_{y}$ respectively, and for certain constants $L_{i}, L_{i 1}, L_{i 2}$ (which may depend on $L_{0}, N_{i}, N_{i 1}, N_{i 2}, N_{0}, G,(B), \Omega$ ). Then the cost functional $I[z, u]$ possesses an absolute minimum in $\Omega$.

Assume now that $G$ is unbounded, open, and of class $K_{01}$, and that $G=\cup G_{N}$, $G_{N} \subset G_{N+1}$, is a typical representation of $G$. Assume that (7) holds as before. If we know that for every pair $z, u$ of $\Omega$ we have

$$
z^{i} \in W_{p i}^{1}(G), \quad i=1, \ldots, n,
$$

and that there are constants $N_{0}, N_{0}^{\prime}, M_{i}^{\prime}$ such that

$$
\begin{gathered}
D_{p_{i}}^{1}\left(z^{i}, G\right) \leqq M_{i}^{\prime}, \quad i=1, \ldots, n, \\
\iint_{G}\left|f_{0}(x, y, z(x, y), u(x, y))\right|^{p_{0}} d x d y \leqq N_{0}, \\
\iint_{G}\left|f_{0}(x, y, z(x, y), u(x, y))\right| d x d y \leqq N_{0}^{\prime}
\end{gathered}
$$

for every admissible pair $z, u$ of the class $\Omega$, then Theorem 2 still holds as above, and for the minimizing admissible pairs $z, u$ of the class $\Omega$ of which we assert the existence (at least one) we know that (11), (12) hold.

Assume again that $G$ is unbounded, open, and of class $K_{01}$, that $G=\bigcup G_{N}$, $G_{N} \subset G_{N+1}$, is a typical representation of $G$ as before, and that (7) holds. Theorem 2 holds also in a particularly weak form. Indeed assume that for every pair $z, u$ of the class $\Omega$ we have $z^{i} \in W_{p_{i}}^{1}\left(G_{N}\right)$ for every $N, p_{i}>1, i=1, \ldots, n$. Assume that relations (10) hold in the weak form

$$
\begin{array}{cc}
\iint_{G_{N}}\left|f_{0}(x, y, z(x, y)), u(x, y)\right|^{p 0} d x d y \leqq N_{0}(N), \\
\iint_{G}\left|f_{0}(x, y, z(x, y), u(x, y))\right| d x d y \leqq N_{0}^{\prime}, \\
\iint_{G_{N}}\left|z^{i}\right|^{p_{i}} d x d y \leqq N_{i}(N) & \text { for } i \in\{\beta\}, \\
\iint_{G_{N}}\left|D_{x} z^{i}\right|^{p} d x d y \leqq N_{i 1}(N) & \text { for } i \in\{\beta\}_{x}, \\
\iint_{G_{N}}\left|D_{y} z^{i}\right|^{p_{i}} d x d y \leqq N_{i 2}(N) & \text { for } i \in\{\beta\}_{y}
\end{array}
$$


for every $N$ and for certain constants $p_{0}>1, N_{0}^{\prime} \geqq 0$, and other constants $N_{0}(N)$, $N_{i}(N), N_{i 1}(N), N_{i 2}(N)$ depending on $N$. Assume that $(z, u) \in \Omega, I[z, u] \leqq L_{0}$ implies

$$
\begin{gathered}
\iint_{G_{N}}\left|z^{i}\right|^{p_{t}} d x d y \leqq L_{i}(N), \\
\iint_{G_{N}}\left|D_{x} z^{i}\right|^{p_{i}} d x d y \leqq L_{i 1}(N), \quad \iint_{G_{N}}\left|D_{y} z^{i}\right|^{p_{i}} d x d y \leqq L_{i 2}(N),
\end{gathered}
$$

for certain constants $L_{i}(N), L_{i 1}(N), L_{i 2}(N)$ (which may depend on $N, L_{0}$, $\left.N_{0}(N), N_{0}^{\prime}, N_{i}(N), N_{i 1}(N), N_{i 2}(N), G,(B), \Omega\right)$ and for all $i=1, \ldots, n$ which are not in $\{\beta\},\{\beta\}_{x},\{\beta\}_{y}$ respectively. Under these weak assumptions Theorem 2 still holds, and for the minimizing admissible pairs $z, u$ of the class $\Omega$ of which we assert the existence (at least one) we know that

$$
\tilde{D}_{p_{i}}^{1}\left(z^{i}, G_{N}\right) \leqq M_{i N}^{\prime}, \quad i=1, \ldots, n,
$$

for every $N$ and constants $M_{i N}^{\prime}$ which may depend on $N$.

Existence Theorem 3. Let $G$ be bounded, open, and of class $K_{01}$, let $A$ be closed, let $U(x, y, z)$ be nonempty and closed for every $(x, y, z) \in A$, and assume that $U(x, y, z)$ satisfies property $(U)$ in $A$. Let $\tilde{f}(x, y, z, u)=\left(f_{0}, f\right)=\left(f_{0}, X_{i}, Y_{i}\right.$, $i=1, \ldots, n)$ be continuous on $M$, and let us assume that the set $\tilde{Q}(x, y, z)$ of all $\tilde{\zeta}=\left(\zeta_{0}, \zeta\right) \in E_{2 n+1}$ with $\zeta_{0} \geqq f_{0}(x, y, z, u), \zeta=f(x, y, z, u), u \in U(x, y, z)$, is a convex closed subset of $E_{2 n+1}$ for every $(x, y, z) \in A$, and that $\tilde{Q}(x, y, z)$ satisfies property $(Q)$ in $A$. Let us assume that $f_{0}(x, y, z, u) \geqq-M_{0}$ for all $(x, y, z, u) \in M$ and some constant $M_{0} \geqq 0$. Let $(B)$ be a system of boundary conditions satisfying property $\left(P_{1}\right)$. Let $\Omega$ be a nonempty complete class of admissible pairs $z(x, y)=$ $\left(z^{1}, \ldots, z^{n}\right), u(x, y)=\left(u^{1}, \ldots, u^{m}\right),(x, y) \in G$, with $z^{i} \in W_{p_{i}}^{1}(G), p_{i}>1, i=1, \ldots, n$, $u^{j}$ measurable in $G, j=1, \ldots, m$, and satisfying given inequalities

$$
\begin{aligned}
\iint_{G}\left|z^{i}\right|^{p_{i}} d x d y \leqq N_{i} & \text { for } i \in\{\beta\}, \\
\iint_{G}\left|D_{x} z^{i}\right|^{p_{i}} d x d y \leqq N_{i 1} & \text { for } i \in\{\beta\}_{x}, \\
\iint_{G}\left|D_{y} z^{i}\right|^{p_{t}} d x d y \leqq N_{i 2} & \text { for } i \in\{\beta\}_{y}
\end{aligned}
$$

for certain constants $N_{i}, N_{i 1}, N_{i 2}$ and all $i$ of certain systems $\{\beta\},\{\beta\}_{x},\{\beta\}_{y}$ of indices $1,2, \ldots, n$ (which may be empty). Assume that $(z, u) \in \Omega, I[z, u] \leqq L_{0}$ implies

$$
\begin{gathered}
\iint_{G}\left|z^{i}\right|^{p_{i}} d x d y \leqq L_{i}, \\
\iint_{G}\left|D_{x} z^{i}\right|^{p_{i}} d x d y \leqq L_{i 1}, \quad \iint_{G}\left|D_{y} z^{i}\right|^{p_{i}} d x d y \leqq L_{i 2},
\end{gathered}
$$

for certain constants $L_{i}, L_{i 1}, L_{i 2}$ (which may depend on $L_{0}, N_{i}, N_{i 1}, N_{i 2}, G$, $(B), \Omega)$ and for all $i=1, \ldots, n$ which are not in $\{\beta\},\{\beta\}_{x},\{\beta\}_{y}$ respectively. Then the cost functional $I[z, u]$ possesses an absolute minimum in $\Omega$.

Assume that $G$ is unbounded, open, and of class $K_{01}$, and that $G=\bigcup G_{N}$, $G_{N} \subset G_{N+1}$, is a typical representation of $G$. Assume that $f_{0}(x, y, z, u) \geqq-\psi(x, y)$ for all $(x, y, z, u) \in M$, where $\psi(x, y) \geqq 0$ is a given $L$-integrable function in $G$. If 
we know that for every pair $z$, $u$ of $\Omega$ we have

$$
z^{i} \in W_{p_{i}}^{1}(G), \quad i=1, \ldots, n,
$$

and that there are constants $M_{i}^{\prime}$ such that

$$
\tilde{D}_{p_{i}}\left(z^{i}, G\right) \leqq M_{i}^{\prime}, \quad i=1, \ldots, n,
$$

for every admissible pair $z$, $u$ of the class $\Omega$, then Theorem 3 still holds as above, and for the minimizing admissible pairs $z, u$ of the class $\Omega$ of which we assert the existence (at least one) we know that (14), (15) hold.

Assume again that $G$ is unbounded, open, and of class $K_{01}$, and that $G=\cup G_{N}$, $G_{N} \subset G_{N+1}$, is a typical representation of $G$ as before. Theorem 3 holds then in a particularly weak form. Indeed assume that for every pair $z$, $u$ of the class $\Omega$ we have $z^{i} \in W_{p_{i}}^{1}\left(G_{N}\right)$ for every $N, p_{i}>1, i=1, \ldots, n$. Assume that $f_{0}(x, y, z, u) \geqq$ $-\psi(x, y)$ where $\psi(x, y) \geqq 0$ is of class $L_{1}(G)$. Assume that relations (13) (if any) hold in the weak form

$$
\begin{aligned}
\iint_{G_{N}}\left|z^{i}\right|^{p_{i}} d x d y \leqq N_{i}(N) & \text { for } i \in\{\beta\}, \\
\iint_{G_{N}}\left|D_{x} z^{i}\right|^{p_{i}} d x d y \leqq N_{i 1}(N) & \text { for } i \in\{\beta\}_{x}, \\
\iint_{G_{N}}\left|D_{y} z^{i}\right|^{p_{i}} d x d y \leqq N_{i 2}(N) & \text { for } i \in\{\beta\}_{y}
\end{aligned}
$$

for every $N$ and for certain constants $N_{i}(N), N_{i 1}(N), N_{i 2}(N)$ which may depend on $N$. Assume that $(z, u) \in \Omega, I[z, u] \leqq L_{0}$ implies

$$
\begin{gathered}
\iint_{G_{N}}\left|z^{i}\right|^{p_{i}} d x d y \leqq L_{i}(N), \\
\iint_{G_{N}}\left|D_{x} z^{i}\right|^{p_{i}} d x d y \leqq L_{i 1}(N), \quad \iint_{G_{N}}\left|D_{y} z^{i}\right|^{p_{i}} d x d y \leqq L_{i 2}(N)
\end{gathered}
$$

for certain constants $L_{i}(N), L_{i 1}(N), L_{i 2}(N)$ (which may depend on $N, L_{0}, N_{i}(N)$, $\left.N_{i 1}(N), N_{i 2}(N), G,(B), \Omega\right)$ and for all $i=1, \ldots, n$ which are not in $\{\beta\},\{\beta\}_{x},\{\beta\}_{y}$ respectively. Under these weak assumptions Theorem 3 still holds, and for the minimizing admissible pairs $z, u$ of the class $\Omega$ of which we assert the existence (at least one) we know that

$$
\tilde{D}_{p_{i}}\left(z^{i}, G_{N}\right) \leqq M_{i N}^{\prime}, \quad i=1, \ldots, n,
$$

for every $N$ and constants $M_{i N}^{\prime}$ which may depend on $N$.

Remark 1. Theorems 1, 2, 3 above are particularizations, for problems with partial differential equations in RASHEVSKY's form, of the existence theorems we have stated and proved in [3], [4], and [5, § 5] for fixed bounded or unbounded domains.

Remark 2. In Theorems 1, 2, 3, as well as in Theorem 4 below, it is enough to require that the boundary conditions $(B)$ satisfy property $\left(P_{1}\right)$ relatively to the complete class $\Omega$ under consideration and for the pairs $z, u$ of the class $\Omega$ for which $I[z, u] \leqq L_{0}$. 
Remark 3. We have shown in [5] by means of examples that, for $G$ unbounded, the requirement $f_{0} \geqq-\psi, \psi \in L_{1}(G)$, cannot be replaced by $f_{0} \geqq-M_{0}, M_{0}$ a positive constant.

\section{§ 4. An Existence Theorem for Lagrange Problems in Sobolev Classes $W_{1}$}

Existence Theorem 4. Let $G$ be bounded, open, and of class $K_{01}$, let $A$ be closed, let $U(x, y, z)$ be nonempty and closed for every $(x, y, z) \in A$, and assume that $U(x, y, z)$ satisfies property $(U)$ in $A$. Let $\tilde{f}(x, y, z, u)=\left(f_{0}, f\right)=\left(f_{0}, X_{i}, Y_{i}\right.$, $i=1, \ldots, n)$ be continuous on $M$, and let us assume that the set $\tilde{Q}(x, y, z)$ of all $\tilde{\zeta}=\left(\zeta^{0}, \zeta\right) \in E_{2 n+1}$ with $\zeta^{0} \geqq f_{0}(x, y, z, u), \zeta=f(x, y, z, u), u \in U(x, y, z)$, is a convex closed subset of $E_{2 n+1}$ for every $(x, y, z) \in A$, and that $Q(x, y, z)$ satisfies property $(Q)$ in $A$. Let us assume that there is a continuous scalar function $\Phi(\xi), 0 \leqq \xi<+\infty$, and two constants $C, D \geqq 0$ such that $\Phi(\xi) / \xi \rightarrow+\infty$ as $\xi \rightarrow+\infty$, and

$$
\begin{array}{r}
f_{0}(x, y, z, u) \geqq \Phi(|u|), \quad\left|X_{i}(x, y, z, u)\right|,\left|Y_{i}(x, y, z, u)\right| \leqq C+D|u| \\
\quad \text { for all }(x, y, z, u) \in M \text { and } i=1, \ldots, n .
\end{array}
$$

Let $(B)$ be a system of boundary conditions satisfying property $\left(P_{1}\right)$. Let $\Omega$ be a nonempty complete class of admissible pairs $z(x, y)=\left(z^{1}, \ldots, z^{n}\right), u(x, y)=$ $\left(u^{1}, \ldots, u^{m}\right),(x, y) \in G, z^{i} \in W_{1}^{1}(G), i=1, \ldots, n, u_{j}$ measurable in $G, j=1, \ldots, m$, satisfying given inequalities

$$
\begin{aligned}
& \iint_{G}\left|z^{i}\right| d x d y \leqq N_{i} \quad \text { for } i \in\{\beta\}, \\
& \iint_{G}\left|D_{x} z^{i}\right| d x d y \leqq N_{i 1} \quad \text { for } i \in\{\beta\}_{x}, \quad \iint_{G}\left|D_{y} z^{i}\right| d x d y \leqq N_{i 2} \quad \text { for } i \in\{\beta\}_{y}
\end{aligned}
$$

for certain constants $N_{i}, N_{i 1}, N_{i 2}$ and all $i$ of certain systems $\{\beta\},\{\beta\}_{x},\{\beta\}_{y}$ of indices $1,2, \ldots, n$ (which may be empty). Assume that $(z, u) \in \Omega, I[z, u] \leqq L_{0}$ implies

$$
\iint_{G}\left|z^{i}\right| d x d y \leqq L_{i}, \quad i=1, \ldots, n,
$$

for certain constants $L_{i}$ (which may depend on $L_{0}, N_{i}, N_{i 1}, N_{i 2}, G,(B), \Omega$ ). Then the cost functional $I[z, u]$ possesses an absolute minimum in $\Omega$.

Assume that $G$ is unbounded, open, and of class $K_{01}$, and that $G=\cup G_{N}$, $G_{N} \subset G_{N+1}$, is a typical representation of $G$. Let us assume (i) that $f_{0}(x, y, z, u) \geqq$ $-\psi(x, y)$ for all $(x, y, z, u) \in M$, where $\psi(x, y) \geqq 0$ is of class $L_{1}(G)$. Assume (ii) that for every $N$ there is a continuous function $\Phi_{N}(\xi), 0 \leqq \xi<+\infty$, and two constants $C_{N}, D_{N} \geqq 0$ such that $\Phi_{N}(\xi) / \xi \rightarrow+\infty$ as $\xi \rightarrow+\infty$, and

$$
\begin{array}{r}
f_{0}(x, y, z, u) \geqq \Phi_{N}(|u|), \quad\left|X_{i}(x, y, z, u)\right|,\left|Y_{i}(x, y, z, u)\right| \leqq C_{N}+D_{N}|u| \\
\quad \text { for all }(x, y, z, u) \in M \text { with }(x, y) \in G_{N} .
\end{array}
$$

Assume that for every pair $z, u$ of the class $\Omega$ we have

$$
z^{i} \in W_{1}^{1}(G), \quad i=1, \ldots, n
$$

and that there are constants $M_{i}^{\prime}$ such that

$$
\tilde{D}_{1}\left(z^{i}, G\right) \leqq M_{i}^{\prime}, \quad i=1, \ldots, n,
$$


for every admissible pair $z, u$ of the class $\Omega$. Then Theorem 4 still holds as above, and for the minimizing admissible pairs $z, u$ of the class $\Omega$ of which we assert the existence (at least one) we know that (19) and (20) hold.

Assume that $G$ is unbounded, open, and of class $K_{01}$ as before, and that $G=\bigcup G_{N}, G_{N} \subset G_{N+1}$, is a typical representation of $G$. Assume that (i) and (ii) hold as before. Theorem 4 holds also in a particularly weak form. Indeed assume that for each pair $z, u$ of the class $\Omega$ we have $z^{i} \in W_{1}^{1}\left(G_{N}\right), i=1, \ldots, n$, for every $N$, and that relations (17) hold in the weak form

$$
\begin{array}{cc}
\iint_{G_{N}}\left|z^{i}\right| d x d y \leqq N_{i}(N) & \text { for } i \in\{\beta\}, \\
\iint_{G_{N}}\left|D_{x} z^{i}\right| d x d y \leqq N_{i 1}(N) & \text { for } i \in\{\beta\}_{x}, \\
\iint_{G_{N}}\left|D_{y} z^{i}\right| d x d y \leqq N_{i 2}(N) & \text { for } i \in\{\beta\}_{y}
\end{array}
$$

where $N_{i}(N), N_{i 1}(N), N_{i 2}(N)$ are given constants which may depend on $N$. Assume that $(z, u) \in \Omega, I[z, u] \leqq L_{0}$ implies

$$
\iint_{G_{N}}\left|z^{i}\right| d x d y \leqq L_{i}(N)
$$

for every $N$, where $L_{i}(N)$ are constants (which may depend on $N, L_{0}, N_{i 1}(N)$, $\left.N_{i 2}(N), G,(B), \Omega\right)$, and for all $i=1, \ldots, n$ which are not in $\{\beta\}$. Then Theorem 4 still holds, and for the minimizing admissible pairs $z, u$ of the class $\Omega$ of which we assert the existence (at least one) we know that

$$
\tilde{D}_{1}\left(z^{i}, G_{N}\right) \leqq M_{i N}^{\prime}, \quad i=1, \ldots, n
$$

for every $N$ and constants $M_{i N}^{\prime}$ which may depend on $N$.

Remark 4. Growth condition (16) can be replaced by the following more general condition: There is a constant $M_{0} \geqq 0$ and, for every $\varepsilon>0$, another constant $M_{\varepsilon} \geqq 0$ such that $f_{0}(x, y, z, u) \geqq-M_{0}$ and $\left|X_{i}(x, y, z, u)\right|,\left|Y_{i}(x, y, z, u)\right| \leqq$ $M_{\varepsilon}+\varepsilon\left[f_{0}(x, y, z, u)+M_{0}\right]$ for every $(x, y, z, u) \in M, i=1, \ldots, n$. Analogously, growth condition (18) can be replaced by a similar condition with constants $M_{0}, M_{\varepsilon}$ replaced by constants $M_{0 N}, M_{\varepsilon N}$ which may depend on $\varepsilon$ (see $[5, \S 6]$ ). The constants $M_{0}, M_{\varepsilon}$ (or $M_{0 N}, M_{\varepsilon N}$ ) can be also replaced by integrable functions $M_{0}(x, y), M_{\varepsilon}(x, y)$. On the other hand, growth condition (16) [or (18)] has implications which make it easier to verify that the sets $\tilde{Q}$ satisfy condition $(Q)$ in $A$. We shall discuss this point elsewhere.

Remark 5. Theorem 4 above is a particularization, for problems with partial differential equations in RASHEVSKY's form, of the existence theorem we have stated and proved in $[5, \S 6]$ for fixed bounded or unbounded domains.

Remark 6. In many cases the sets $\tilde{Q}$ or $\tilde{Q}$ are not convex, and examples show that an optimal solution may fail to exist. In these cases, it has been proposed to replace the system and the functional

$$
\begin{gathered}
z_{x}^{i}=X_{i}(x, y, z, u), \quad z_{y}^{i}=Y_{i}(x, y, z, u), \\
I=\iint_{G} f_{0}(x, y, z, u) d x d y
\end{gathered}
$$


by a new system and a new functional

$$
\begin{gathered}
z_{x}^{i}=\sum \lambda_{j} X_{i}\left(x, y, z, u^{(j)}\right), \quad z_{y}^{i}=\sum \lambda_{j} Y_{i}\left(x, y, z, u^{(j)}\right), \\
J=\iint_{G} \sum \lambda_{j} f_{0}\left(x, y, z, u^{(j)}\right) d x d y,
\end{gathered}
$$

where $\sum$ ranges from 1 to $\mu$, where we still have $n$ state variables $z^{1}, \ldots, z^{n}$, but the control variable, or $m$-vector $u$, is now replaced by a new control variable, or $(\mu m+\mu)$-vector $v=\left(u^{(1)}, \ldots, u^{(\mu)}, \lambda_{1}, \ldots, \lambda_{\mu}\right)$ (GAMKRELIDZE's sliding regimes, or weak solutions). Here each $m$-vector $u^{(j)}$ is subject to the same constraint as before, or $u^{(j)} \in U(x, y, z), j=1, \ldots, \mu$, while the $\mu$ scalars $\lambda_{j}$ are subject to the limitations $\lambda_{j} \geqq 0, \sum \lambda_{j}=1$. In other words, if $\Gamma$ denotes the simplex $\lambda_{j} \geqq 0$, $j=1, \ldots, \mu, \sum \lambda_{j}=1$, we require $v \in V(x, y, z)=U^{\mu} \times \Gamma$. We shall require the state variables $z^{i}$ to belong to the same Sobolev spaces $W_{p i}^{1}(G)$ as before, and we shall require all variables $u^{(j)}, \lambda_{j}, j=1, \ldots, \mu$, to be measurable. Interpreting the $\lambda_{j}$ as probability distributions, the new state variables $z^{i}$ can be thought of as generated by a probability distribution of the $\mu$ controls $u^{(j)}$ (acting contemporaneously). Here the sets $\tilde{Q}(x, y, z)$ shall be replaced by analogous sets $\tilde{Q}^{*}(x, y, z)$. Here $\widetilde{Q}^{*}(x, y, z)$ is the set of all points $\zeta=\left(\zeta_{0}, \ldots, \zeta_{2 n}\right)$, with

$$
\begin{gathered}
\zeta_{0} \geqq \sum \lambda_{j} f_{0}\left(x, y, z, u^{(j)}\right), \\
\zeta_{i}=\sum \lambda_{j} X_{i}\left(x, y, z, u^{(j)}\right), \quad \zeta_{n+i}=\sum \lambda_{j} Y_{i}\left(x, y, z, u^{(j)}\right), \quad i=1, \ldots, n,
\end{gathered}
$$

and hence, each point of $\tilde{Q}^{*}$ can be thought of as the convex combination of $\mu$ points of the set $\tilde{Q}$. Thus, if $\mu \geqq 2 n+2$, the sets $\tilde{Q}^{*}$ are all convex. For more details see [5], and, for $v=1$, see $[1,2]$.

Remark 7. Note that growth condition (16) for system (21) does not imply an analogous condition for system (22). Nevertheless, relation $\left|X_{i}\right|,\left|Y_{i}\right| \leqq M_{\varepsilon}+$ $\varepsilon\left(f_{0}+M_{0}\right)$ of Remark 4 implies an analogous relation for system (22). (See $[5, \S 7]$.)

\section{Part II.}

\section{§ 5. Lurie's Optimization Problems \\ in the Theory of Magnetohydrodynamical Channel Flow}

In harmony with LURIE $[7,8]$ let us consider the rectilinear motion

$$
\{\vec{v}=[V(y), 0,0]\}, \quad(x, y) \in G=[-\infty<x<+\infty,-d<y<d],
$$

of a conducting fluid along a plane channel of width $2 d$. Let the specific resistance $u(x, y)$ of the fluid be restricted by constants $a$ and $b$, so that $0<a \leqq u(x, y) \leqq$ $b<+\infty$. Let $E$ and $H$ denote the electric and magnetic field respectively. The walls of the channel will be assumed insulating everywhere except for two sections of equal length $2 l$ occupied by ideally conducting electrodes located opposite each other on different walls. The electrodes are connected through the outer load $R$. As soon as the transverse magnetic field $\vec{B}=-i_{3} B(x)$ is imposed on the moving fluid, an electric current of density $\vec{j}=\left(j_{x}, j_{y}, 0\right)$ is induced inside the channel. We shall use the notation

$$
j_{x}=v, \quad j_{y}=w, \quad v=v(x, y), \quad w=w(x, y) .
$$


Then through the outer load there flows a total current equal to

$$
I=\int_{-l}^{+l} w(x, \pm \delta) d x
$$

Provided the magnetic Reynolds number $\left(\operatorname{Re}_{m}\right)$ is small compared with unity, the induced magnetic field can be neglected in comparison with the external field $[7,8]$, that is, we can take $\vec{B}=\vec{H}$. Moreover, if the magnetohydrodynamic (mhd) parameter of interaction $(N)$ is also small, it is possible to neglect the Lorentz force in

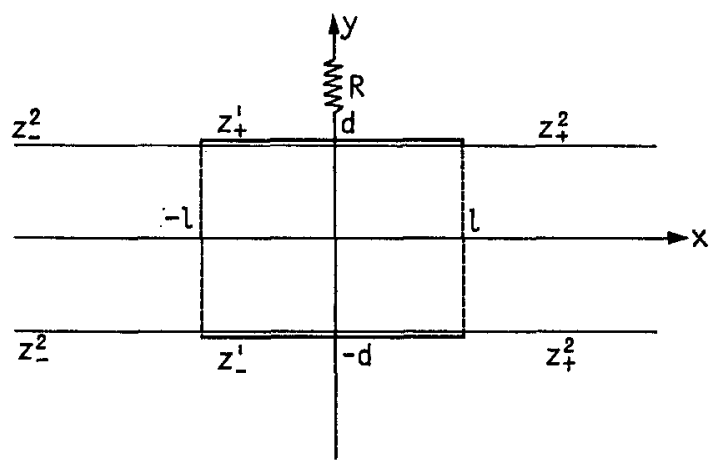

Fig. 1

the dynamic equation so that the velocity distribution can be considered as prescribed by the purely hydrodynamical problem of rectilinear motion in a channel $[7,8]$. These two assumptions simplify the basic mhd equations, as given, for instance, in [11]. By introducing the notations

$$
\vec{j}=-\operatorname{curl} \vec{i}_{3} z^{2}, \quad \vec{E}=-\operatorname{grad} z^{1}, \quad z^{1}=z^{1}(x, y), \quad z^{2}=z^{2}(x, y),
$$

so that $z^{1}$ and $z^{2}$ are the electrical potential and current functions respectively, LURIE $[7,8]$ gives the mhd equations in the form

$$
\begin{array}{ll}
\frac{\partial z^{1}}{\partial x}=-u v, & \frac{\partial z^{1}}{\partial y}=-u w+c^{-1} V B, \\
\frac{\partial z^{2}}{\partial x}=w, & \frac{\partial z^{2}}{\partial y}=-v,
\end{array}
$$

where $c$ is a positive constant, and to these equations should also be added the compatibility relations

$$
\frac{\partial}{\partial y}\left(\frac{\partial z^{1}}{\partial x}\right)=\frac{\partial}{\partial x}\left(\frac{\partial z^{1}}{\partial y}\right), \quad \frac{\partial}{\partial y}\left(\frac{\partial z^{2}}{\partial x}\right)=\frac{\partial}{\partial x}\left(\frac{\partial z^{2}}{\partial y}\right)
$$

which, after reduction, become

$$
\frac{\partial}{\partial x}\left(c^{-1} V B-u w\right)+\frac{\partial}{\partial y}(u v)=0, \quad \frac{\partial w}{\partial x}+\frac{\partial w}{\partial y}=0,
$$

and where $a \leqq u(x, y) \leqq b$. The upper limit $b$ corresponds to the resistance of the fluid when all external ionization factors are withdrawn, the lower limit characterizes the maximum number of ionization possibilities. 
At infinity, the components $v, w$ of the current density should be assumed to vanish. Because of equations (4) the functional $I$ takes the form

$$
I=z^{2}(l, \pm d)-z^{2}(-l, \pm d) .
$$

The boundary conditions, as proposed in $[7,8]$, are as follows

$$
\begin{gathered}
z^{1}(x, \pm d)=z_{ \pm}^{1}=\text { constant for }|x| \leqq l, \\
z^{2}(x, \pm d)=z_{+}^{2}=\text { constant for } l<x<+\infty, \\
z^{2}(x, \pm d)=z_{-}^{2}=\text { constant for }-\infty<x<-l, \\
z^{1}(+\infty, d)-z^{1}(+\infty,-d)=z^{1}(-\infty, d)-z^{1}(-\infty,-d)=c^{-1} \int_{-d}^{d} B(x) V(y) d y=e, \\
z^{2}(+\infty, \pm d)-z^{2}(-\infty, \pm d)=R^{-1}\left(z_{+}^{1}-z_{-}^{1}\right) .
\end{gathered}
$$

The last equation is only a relation between given constants and can be written in the form

$$
z_{+}^{1}-z_{-}^{1}=R\left(z_{+}^{2}-z_{-}^{2}\right)
$$

The total Joule losses are given in [7] in the form

$$
J=\int_{-\infty}^{+\infty} \int_{-d}^{d} u\left(v^{2}+w^{2}\right) d x d y
$$

For the case of an homogeneous magnetic field $B$, the functionals $I$ and $J$ are related by the identity $J=I e-I^{2} R$, as stated in [7]. LURIE's problems for the mhd channel flow in $[7,8]$ are the problem of the maximum of $I$ (maximum current), and the problem of the minimum of $J$ (minimum Joule losses). As LURIE mentions, other analogous problems can be taken into consideration. We shall consider below the problem of the minimum of $J$.

\section{The Lagrange-Type Problem of LURIE}

If we introduce the notation

$$
W(y)=c^{-1} \int_{-d}^{y} V(y) d y, \quad-d \leqq y \leqq d,
$$

and the new variable $Z=\left(Z^{1}, Z^{2}\right)$ by means of the relations

$Z^{1}(x, y)=z^{1}(x, y)-\int_{-d}^{y} c^{-1} V(y) B(x) d y=z^{1}(x, y)-W(y) B(x), Z^{2}(x, y)=z^{2}(x, y)$, then equations (4) become

$$
\begin{array}{ll}
\frac{\partial Z^{1}}{\partial x}=-u v-W(y) B^{\prime}(x), & \frac{\partial Z^{1}}{\partial y}=-u w, \\
\frac{\partial Z^{2}}{\partial x}=w, & \frac{\partial Z^{2}}{\partial y}=-v,
\end{array}
$$

with $-\infty<x<+\infty,-d<y<d$, while the accompanying relations (5) remain unchanged, and where $B^{\prime}=d B / d x$. 
If $G$ denotes the unbounded open set $G=[-\infty<x<+\infty,-d<y<d]$, we shall consider the vector functions $Z(x, y)=\left(Z^{1}, Z^{2}\right)$, and scalar functions $u(x, y)$, $v(x, y), w(x, y)$, satisfying $(a)$ the first order partial differential equations (8) (of RASHEVKY's type); (b) the associated relations analogous to (5) which we shall write in the weak form

$$
\iint_{G}\left(Z_{x}^{i} \varphi_{y}-Z_{y}^{i} \varphi_{x}\right) d x d y=0, \quad i=1,2,
$$

for every $\varphi \in C_{0}^{\infty}(G) ;(c)$ the constraints

$$
\begin{gathered}
0<a \leqq u(x, y) \leqq b<+\infty, \\
\iint_{G} u\left(v^{2}+w^{2}\right) d x d y<+\infty,
\end{gathered}
$$

(d) the boundary conditions

$$
\begin{aligned}
& Z^{1}(x,-d)=z_{-}^{1}, \quad Z^{1}(x, d)=z_{+}^{1}-W(d) B(x) \quad \text { for }|x| \leqq l, \\
& Z^{2}(x, \pm d)=z_{+}^{2} \text { for } l<x<+\infty, \quad Z^{2}(x, \pm d)=z_{-}^{2} \text { for }-\infty<x<-l, \\
& Z^{1}(+\infty, d)=Z^{1}(+\infty,-d), \quad Z^{1}(-\infty, d)=Z^{1}(-\infty,-d) .
\end{aligned}
$$

Since we are interested in the minimum of $J$, it is not restrictive to add the further constraint

$$
J=\int_{-\infty}^{+\infty} \int_{-d}^{d} u\left(v^{2}+w^{2}\right) d x d y \leqq M
$$

for some sufficiently large constant $M$. Here $R, d, l, a, b, c, M$ are positive constants, $z_{-}^{1}, z_{+}^{1}, z_{+}^{2}, z_{-}^{2}$ are constants such that $z_{+}^{1}-z_{-}^{1}=R\left(z_{+}^{2}-z_{-}^{2}\right), V(y), B(x)$ are given functions, $B(x)$ continuous with $B^{\prime}(x)$, and $W(y)$ is defined by (7).

We shall assume $|V(y)| \leqq c D_{1}$ for $-d \leqq y \leqq d$ and some constant $D_{1}$. We shall also assume $B^{\prime} \in L_{2}(-\infty,+\infty)$ and we denote by $D_{2}$ the constant

$$
D_{2}=\int_{-\infty}^{+\infty} B^{\prime 2}(x) d x
$$

so that $|W(y)| \leqq 2 d D_{1}$. Then from (8) and (12) follows

$$
\begin{aligned}
& \left.\iint_{G}\left|\partial Z^{1}\right| \partial x\right|^{2} d x d y \leqq 2 b M+16 d^{3} D_{1}^{2} D_{2}, \\
& \left.\iint_{G}\left|\partial Z^{1}\right| \partial y\right|^{2} d x d y \leqq b M, \\
& \left.\iint_{G}\left|\partial Z^{2}\right| \partial x\right|^{2} d x d y \leqq a^{-1} M,\left.\quad \iint_{G}\left|\partial Z^{2}\right| \partial y\right|^{2} d x d y \leqq a^{-1} M .
\end{aligned}
$$

Thus, for any pair $Z=\left(Z^{1}, Z^{2}\right), U=(u, v, w)$ satisfying $(a),(b),(c),(d)$ and (12) we necessarily have $a \leqq u \leqq b,(v, w) \in E_{2}, Z_{x}^{1}, Z_{y}^{1}, Z_{x}^{2}, Z_{y}^{2} \in L_{2}(G)$.

Remark 8. With the notations of $\S 3, f, f_{0}$ and $\tilde{f}=\left(f_{0}, f\right)$ are now given by

$$
f(x, y, u, v, w)=\left(-u v-W(y) B^{\prime}(x),-u w, w,-v\right), \quad f_{0}=u\left(v^{2}+w^{2}\right),
$$


and $U$ is the fixed set $U=[a \leqq u \leqq b,-\infty<v, w<+\infty]$. Then the corresponding sets $\tilde{Q}(x, y)$ and $\tilde{Q}(x, y)=\tilde{f}(x, y, U)$ are not convex since their common projections on the $\zeta^{1} \zeta^{2} \zeta^{3} \zeta^{4}$-space are the sets $Q(x, y)=f(x, y, U)$ of all $\left(\zeta^{1}, \zeta^{2}, \zeta^{3}, \zeta^{4}\right)$ such that

$$
\zeta^{1}=-u v-W(y) B^{\prime}(x), \quad \zeta^{2}=-u w, \quad \zeta^{3}=w, \quad \zeta^{4}=-v, \quad(u, v, w) \in U .
$$

It is enough to show that their displacement $Q_{0}$ is not convex, where $Q_{0}$ is the set of all $\zeta=\left(\zeta^{1}, \zeta^{2}, \zeta^{3}, \zeta^{4}\right)$ with

$$
\zeta^{1}=-u v, \quad \zeta^{2}=-u w, \quad \zeta^{3}=w, \quad \zeta^{4}=-v, \quad(u, v, w) \in U .
$$

For $u=b, v=1, w=1$, we have the point $\zeta_{1}=(-b,-b, 1,-1) \in Q_{0}$, and for $u=a$, $v=-1, w=-1$ we have the point $\zeta_{2}=(a, a,-1,1) \in Q_{0}$, while the point

$$
\zeta=2^{-1} \zeta_{1}+2^{-1} \zeta_{2}=\left[2^{-1}(-b+a), 2^{-1}(-b+a), 0,0\right]
$$

is the image of no point of $U$ since $\zeta^{3}=\zeta^{4}=0$ implies $w=v=0$ and hence $\zeta^{1}=\zeta^{2}=0$, while $-b+a \neq 0$. Thus $\zeta_{1} \in Q_{0}, \zeta_{2} \in Q_{0}, \zeta=2^{-1} \zeta_{1}+2^{-1} \zeta_{2} \notin Q_{0}$, and $Q_{0}$ is not convex.

\section{A Weak Form of LURIE's Optimization Problem}

The weak form of LURIE's optimization problem, corresponding to GAMKRELIDZE's sliding regimes, is considered in this section.

We shall consider a class $\Omega$ of systems $Z^{1}, Z^{2}, u_{j}, v_{j}, w_{j}, \lambda_{j}, j=1, \ldots, \mu$, of functions in $G$, where as before $Z^{1}, Z^{2}$ are the state variables, and $u_{j}, v_{j}, w_{j}, \lambda_{j}$, $j=1, \ldots, \mu$, are new control variables. The variables $\lambda_{j}$ represent probabilistic distributions, and therefore they satisfy the constraints

$$
\left(\lambda_{1}, \ldots, \lambda_{\mu}\right) \in \Gamma=\left[\lambda_{j} \geqq 0, j=1, \ldots, \mu, \lambda_{1}+\cdots+\lambda_{\mu}=1\right] .
$$

For every $j=1, \ldots, \mu$, the triple $\left(u_{j}, v_{j}, w_{j}\right)$ is required to lie in $U$, or $\left(u_{j}, v_{j}, w_{j}\right) \in U$, $j=1, \ldots, \mu$, so that $a \leqq u_{j} \leqq b,\left(v_{j}, w_{j}\right) \in E_{2}$. In other words, the control space is now the set $V=U^{\mu} \times \Gamma$, and the new control variable $\left(u_{j}, v_{j}, w_{j}, \lambda_{j}, j=1, \ldots, \mu\right)$ is required to lie in $U^{\mu} \times \Gamma$.

We shall replace the differential system (8) by the relaxed system

$$
\begin{array}{ll}
Z_{x}^{1}=-\sum \lambda_{j} u_{j} v_{j}-W(y) B^{\prime}(x), & Z_{y}^{1}=-\sum \lambda_{j} u_{j} w_{j}, \\
Z_{x}^{2}=\sum \lambda_{j} w_{j}, & Z_{y}^{2}=-\sum \lambda_{j} v_{j},
\end{array}
$$

where $\sum$ ranges over all $j=1, \ldots, \mu$. The new functional shall now be written in the form

$$
J=\iint_{G} \sum \lambda_{j} u_{j}\left(v_{j}^{2}+w_{j}^{2}\right) d x d y,
$$

and since we seek the minimum of $J$, it is not restrictive to assume that

$$
\iint_{G} \sum_{j} \lambda_{j} u_{j}\left(v_{j}^{2}+w_{j}^{2}\right) d x d y \leqq M
$$

for some sufficiently large constant $M$. The open unbounded set

$$
G=[-\infty<x<+\infty,-d<y<d]
$$

7 Arch. Rational Mech. Anal., Vol. 29 
is obviously of class $K_{01}$, and for its typical representation we shall take $G=\bigcup G_{N}$, $G_{N} \subset G_{N+1}$, with $G_{N}=[-N l<x<N l,-d<y<d], N=1,2, \ldots$.

We shall define the class $\Omega$ as the class of all systems

(16) $Z^{1}(x, y), \quad Z^{2}(x, y), \quad u_{j}(x, y), \quad v_{j}(x, y), \quad w_{j}(x, y), \quad \lambda_{j}(x, y), j=1, \ldots, \mu$, with $Z^{1}, Z^{2} \in W_{2}^{1}\left(G_{N}\right)$ for every $N, u_{j}, v_{j}, w_{j}, \lambda_{j}$ measurable in $G, j=1, \ldots, \mu$, satisfying (15) and the differential equations (13) a.e. in $G$, as well as the constraints mentioned above, or

$$
\left(u_{j}, v_{j}, w_{j}, \lambda_{j}, j=1, \ldots, \mu\right) \in U^{\mu} \times \Gamma,
$$

while for the values of the pairs $\left(Z^{1}, Z^{2}\right)$ we propose no constraint at all, that is, we take all sets $A(x, y)=E_{2}$, and hence $A=(\operatorname{cl} G) \times E_{2}$, a closed subset of the $x y z^{1} z^{2}$-space. We do not require $L_{1}$ or $L_{2}$ integrability of any single function $v_{j}, w_{j}$, $j=1, \ldots, v$, but only the $L_{1}$-integrability of the expression

$$
F_{0}=\sum \lambda_{j} u_{j}\left(v_{j}^{2}+w_{j}^{2}\right)
$$

in $G$ as is implied by the constraint (15). Nevertheless the local $L$-integrability of the functions $u_{j}, \lambda_{j}$ is implied by their measurability and boundedness. Also, we shall restrict $\Omega$ by imposing boundary conditions. We shall impose only the condition (10), or

$$
\begin{aligned}
& Z^{1}(x,-d)=z_{-}^{1}, \quad Z^{1}(x, d)=z_{+}^{1}-W(d) B(x) \text { for }|x|<l, \\
& Z^{2}(x, \pm d)=z_{+}^{2} \text { for } l<x<+\infty, \quad Z^{2}(x, \pm d)=z_{-}^{2} \text { for }-\infty<x<-l .
\end{aligned}
$$

Indeed these conditions possess property $\left(P_{1}\right)$ as mentioned in $\S 1$. A remark concerning condition (11) will follow.

Remark 9. We shall assume that the class $\Omega$ is not empty, that is, we assume that the differential system, the boundary conditions, and the various constraints can be satisfied by some pair $z(x, y), u(x, y)$ as above. This question must be discussed elsewhere, though the elliptic character of the fundamental system (8) hints at the possibility of answering this question in the affirmative.

Let us prove that for any system (16) of the class $\Omega$ we have

$$
Z_{x}^{1}, Z_{x}^{2}, Z_{y}^{1}, Z_{y}^{2} \in L_{2}(G)
$$

Since $Z^{1}, Z^{2} \in W_{2}^{1}\left(G_{N}\right)$ for every $N$, certainly we have $Z_{x}^{1}, Z_{x}^{2}, Z_{y}^{1}, Z_{y}^{2} \in L_{2}\left(G_{N}\right)$ for every $N$. Hence

$$
\iint_{G_{N}}\left(Z_{y}^{1}\right)^{2} d x d y=\iint_{G_{N}}\left(\sum \lambda_{j} u_{j} w_{j}\right)^{2} d x d y .
$$

For every $(x, y) \in G_{N}$, let $s$ be one of the indices $j=1, \ldots, \mu$, for which

so that

$$
\left|\lambda_{s} u_{s} w_{s}\right|=\max _{j=1, \ldots, v}\left|\lambda_{j} u_{j} w_{j}\right|,
$$

and hence, since $a \leqq u_{s} \leqq b$,

$$
\left|\sum \lambda_{j} u_{j} w_{j}\right| \leqq \mu\left|\lambda_{s} u_{s} w_{s}\right|
$$

$$
\iint_{G_{N}}\left(Z_{y}^{1}\right)^{2} d x d y \leqq \mu^{2} \iint_{G_{N}}\left(\lambda_{s} u_{s} w_{s}\right)^{2} d x d y \leqq \mu^{2} b \iint_{G} \lambda_{s}^{2} u_{s} w_{s}^{2} d x d y .
$$


Since $0 \leqq \lambda_{s} \leqq 1$ (hence $0 \leqq \lambda_{s}^{2} \leqq \lambda_{s} \leqq 1$ ), we have also

$$
\begin{gathered}
\iint_{G_{N}}\left(Z_{y}^{1}\right)^{2} d x d y \leqq \mu^{2} b \iint_{G_{N}} \lambda_{s} u_{s} w_{s}^{2} d x d y \leqq \mu^{2} b \iint_{G_{N}} \sum \lambda_{j} u_{j}\left(v_{j}^{2}+w_{j}^{2}\right) d x d y, \\
\iint_{G_{N}}\left(Z_{y}^{1}\right)^{2} d x d y \leqq \mu^{2} b M
\end{gathered}
$$

for every $N$, and hence $Z_{y}^{1} \in L_{2}(G)$, and

$$
\iint_{G}\left(Z_{y}^{1}\right)^{2} d x d y \leqq \mu^{2} b M .
$$

Analogously, we can prove, using the elementary inequality $(\alpha+\beta)^{2} \leqq 2 \alpha^{2}+2 \beta^{2}$, that

$$
\iint_{G_{N}}\left(Z_{x}^{1}\right)^{2} d x d y \leqq 2 \iint_{G_{N}}\left(\sum \lambda_{j} u_{j} v_{j}\right)^{2} d x d y+2 \iint_{G_{N}} W^{2}(y) B^{\prime 2}(x) d x,
$$

and hence, as before

$$
\begin{aligned}
\iint_{G}\left(Z_{x}^{1}\right)^{2} d x d y & \leqq 2 \mu^{2} b \iint_{G} \sum \lambda_{j} u_{j}\left(v_{j}^{2}+w_{j}^{2}\right) d x d y+2 \iint_{G} W^{2}(y) B^{\prime 2}(x) d x d y \\
& \leqq 2 \mu^{2} b M+16 d^{3} D_{1}^{2} D_{2} .
\end{aligned}
$$

By analogous argument we have also

$$
\iint_{G}\left(Z_{x}^{2}\right)^{2} d x d y \leqq \mu^{2} a^{-1} M, \quad \iint_{G}\left(Z_{y}^{2}\right)^{2} d x d y \leqq \mu^{2} a^{-1} M .
$$

Statement (19) is thereby proved.

Remark 10. The $L_{2}$-integrability of $Z_{x}^{1}, Z_{y}^{1}$ which we have just proved certainly assures for $Z^{1}$ a remarkable "smoothness" at $\infty$ in the strip $G$, but does not assure the existence of any of the limits $Z^{1}(+\infty, y), Z^{1}(-\infty, y),-d \leqq y \leqq d$, and even less the existence of the limits $L_{1}, L_{2}$ of $Z^{1}$ as $x \rightarrow+\infty$, or $x \rightarrow-\infty$. (The $L_{1^{-}}$ integrability of $Z_{x}^{1}, Z_{y}^{1}$ in $G$ would assure this.) Essentially for the same reasons the boundary conditions (11) do not satisfy property $\left(P_{1}\right)$ for $p=2$. Thus, this boundary condition has to be abandoned in the present scheme. The question whether the optimal solution has this property shall not be discussed here, and is left to the regularization problem.

In order to apply Existence Theorem 3 we shall now prove that the systems (16) of the class $\Omega$ satisfy uniform integral relations

$$
\iint_{G_{N}}\left(Z^{1}\right)^{2} d x d y \leqq C_{N}, \quad \iint_{G_{N}}\left(Z^{2}\right)^{2} d x d y \leqq D_{N},
$$

where $C_{N}, D_{N}$ are constants which may depend on $N$ (and on the data of the problem), but do not depend on the particular system (16) of the class $\Omega$ taken into consideration.

To prove the first of relations (23) we shall use exclusively relations (20) and (21) which we shall write here in the form

$$
\iint_{G_{N}}\left(Z_{y}^{1}\right)^{2} d x d y \leqq A=\mu^{2} b M, \quad \iint_{G_{N}}\left(Z_{x}^{1}\right)^{2} d x d y \leqq B=2 \mu^{2} b M+16 d^{3} D_{1}^{2} D_{2},
$$

for all $N$. 
It is enough to prove the first relation (23) for functions $Z^{1}$ of class $C^{1}$ in $G_{N}$ satisfying (24), since every function of class $W_{2}^{1}\left(G_{N}\right)$ in a given $G_{N}$, can be approached by means of functions of class $C^{1}$ with the same bounds (24). (This argument has been used already in $[5, \S 2,(2 . i)]$.) Since

$$
\int_{G_{1}}\left(Z_{y}^{1}\right)^{2} d x d y=\int_{-1}^{l} d x \int_{-d}^{d}\left(Z_{y}^{1}\right)^{2} d y \leqq A,
$$

there is at least one point $\bar{x},-l<\bar{x}<l$, such that

$$
\int_{-d}^{d}\left(Z_{y}^{1}(\bar{x}, y)\right)^{2} d y \leqq \frac{A}{2 l} .
$$

Since $Z^{1}(x,-d)=z_{-}^{1}$, a constant, we also have, for $-d \leqq y \leqq d$,

$$
\begin{aligned}
\left|Z^{1}(\bar{x}, y)\right| & =\left|z_{-}^{1}+\int_{-d}^{y} Z_{y}^{1} d y\right| \leqq\left|z_{-}^{1}\right|+\left(\int_{-d}^{y} d y\right)^{\frac{1}{2}}\left(\int_{-d}^{y}\left(Z_{y}^{1}\right)^{2} d y\right)^{\frac{1}{2}} \\
& \leqq\left|z_{-}^{1}\right|+(2 d)^{\frac{1}{2}}(A / 2 l)^{\frac{1}{2}}=A^{\prime},
\end{aligned}
$$

where we have denoted by $A^{\prime}$ the last constant. Now, for $\bar{x} \leqq x \leqq N l, N>1$, we have $x-\bar{x} \leqq N l+l=(N+1) l$, and

and finally

$$
\begin{aligned}
\left|Z^{1}(x, y)\right| & =\left|Z^{1}(\bar{x}, y)+\int_{\bar{x}}^{x} Z_{x}^{1}(\alpha, y) d \alpha\right| \leqq A^{\prime}+\int_{\bar{x}}^{x}\left|Z_{x}^{1}(\alpha, y)\right| d \alpha \\
& \leqq A^{\prime}+\left(\int_{\bar{x}}^{x} d \alpha\right)^{\frac{1}{2}}\left(\int_{\bar{x}}^{x}\left(Z_{x}^{1}(\alpha, y)\right)^{2} d \alpha\right)^{\frac{1}{2}} \\
& \leqq A^{\prime}+(N+1)^{\frac{1}{2}} l^{\frac{1}{2}}\left(\int_{\bar{x}}^{N l}\left(Z_{x}^{1}(\alpha, y)\right)^{2} d \alpha\right)^{\frac{1}{2}},
\end{aligned}
$$

$$
\begin{aligned}
\int_{\bar{x}}^{N l} \int_{-d}^{d}\left(Z^{1}(x, y)\right)^{2} d x d y & \leqq \int_{\bar{x}}^{N l} \int_{-d}^{d}\left[2 A^{\prime 2}+2 l(N+1) \int_{\bar{x}}^{N l}\left(Z_{x}^{1}(\alpha, y)\right)^{2} d \alpha\right] d x d y \\
& \leqq 4 d l(N+1) A^{\prime 2}+2(N+1) l \int_{\bar{x}}^{N l} \int_{-d}^{d} \int_{\bar{x}}^{N l}\left(Z_{x}^{1}(\alpha, y)\right)^{2} d \alpha d x d y \\
& =4 d l(N+1) A^{\prime 2}+2 l^{2}(N+1)^{2} \int_{\bar{x}}^{N l} \int_{-d}^{d}\left(Z_{x}^{1}(x, y)\right)^{2} d x d y \\
& \leqq 4 d l(N+1) A^{\prime 2}+2 l^{2}(N+1)^{2} B .
\end{aligned}
$$

An analogous relation holds for $-N l \leqq x \leqq \bar{x}$, and hence

$$
\iint_{G_{N}}\left(Z^{1}(x, y)\right)^{2} d x d y \leqq 8 d l(N+1) A^{\prime 2}+4 l^{2}(N+1)^{2} B=C_{N} .
$$

The first relation (23) is thereby proved. The remaining relation (23) can be proved by an analogous argument by using inequalities (22) and the information that $Z^{2}(x,-d)=z_{+}^{2}$, a constant, for all $x$ with $l \leqq x \leqq 2 l$. 
If we denote by $u^{*}$ the set of variables $u_{j}, v_{j}, w_{j}, \lambda_{j}, j=1, \ldots, \mu$, we shall now consider (as in the Remark 8) the 4-vector $F$, and the 5-vector $\widetilde{F}=\left(F_{0}, F\right)$ defined by

$$
F\left(x, y, u^{*}\right)=\left(-\sum \lambda_{j} u_{j} v_{j}-W(y) B^{\prime}(x),-\sum \lambda_{j} u_{j} w_{j}, \sum \lambda_{j} w_{j},-\sum \lambda_{j} v_{j}\right),
$$

and $F_{0}$ already defined in (17), or

$$
F_{0}\left(x, y, u^{*}\right)=\sum \lambda_{j} u_{j}\left(v_{j}^{2}+w_{j}^{2}\right) .
$$

We shall now consider first the set $Q(x, y)$ of all $\zeta=\left(\zeta^{0}, \zeta^{1}, \zeta^{2}, \zeta^{3}, \zeta^{4}\right)$ with

$$
\zeta^{0}=u\left(v^{2}+w^{2}\right), \quad \zeta^{1}=-u v-W(y) B^{\prime}(x), \quad \zeta^{2}=-u w, \quad \zeta^{3}=w, \quad \zeta^{4}=-v,
$$

for $(u, v, w) \in U$, and then the set $Q^{*}(x, y)$ of all $\zeta=\left(\zeta^{0}, \ldots, \zeta^{4}\right)$ with

$$
\begin{aligned}
& \zeta^{0}=\sum \lambda_{j} u_{j}\left(v_{j}^{2}+w_{j}^{2}\right), \quad \zeta^{1}=-\sum \lambda_{j} u_{j} v_{j}-W(y) B^{\prime}(x), \\
& \zeta^{2}=-\sum \lambda_{j} u_{j} w_{j}, \quad \zeta^{3}=\sum \lambda_{j} w_{j}, \quad \zeta^{4}=-\sum \lambda_{j} v_{j},
\end{aligned}
$$

with $u^{*}=\left(u_{j}, v_{j}, w_{j}, \lambda_{j}, j=1, \ldots, \mu\right) \in U^{\mu} \times \Gamma$. Thus, $Q^{*}$ is the set of all convex combinations of $\mu$ points arbitrarily taken in $Q$. If we assume $\mu=6$, then certainly the set $Q^{*}(x, y)$ is convex, and this holds for every $(x, y) \in G$ (and even for every $(x, y) \in \mathrm{cl} G$, and hence for every $(x, y, z) \in A \in \mathrm{cl} G \times E_{2}$, since $Q$ does not depend on $z$ ).

As a consequence, the set $\tilde{Q}^{*}(x, y)$ that we obtain, in harmony with Theorem 3 , by replacing the first relation (25) with $\zeta^{0} \geqq \sum \lambda_{j} u_{j}\left(v_{j}^{2}+w_{j}^{2}\right)$, is also convex.

Let us prove that the sets $Q^{*}(x, y)$ are closed. It is enough to prove that the set $Q_{0}$ below, obtained by a rigid displacement in $E_{5}$, is closed. In other words, we shall prove the closure of the set $Q_{0}$ defined as the set of all points $\zeta=\left(\zeta^{0}, \ldots, \zeta^{4}\right)$ such that

$$
\begin{aligned}
& \zeta^{0} \geqq \sum \lambda_{j} u_{j}\left(v_{j}^{2}+w_{j}^{2}\right), \quad \zeta^{1}=-\sum \lambda_{j} u_{j} v_{j}, \\
& \zeta^{2}=-\sum \lambda_{j} u_{j} w_{j}, \quad \zeta^{3}=\sum \lambda_{j} w_{j}, \quad \zeta^{4}=-\sum \lambda_{j} v_{j},
\end{aligned}
$$

with $u^{*}=\left(u_{j}, v_{j}, w_{j}, \lambda_{j}, j=1, \ldots, \mu\right) \in U^{\mu} \times \Gamma$.

Let $\zeta=\left(\zeta^{0}, \ldots, \zeta^{4}\right) \in \operatorname{cl} Q_{0}$, and let $\zeta_{k}=\left(\zeta_{k}^{0}, \ldots, \zeta_{k}^{4}\right), k=1,2, \ldots$, be a sequence of points of $Q_{0}$ convergent to $\zeta$. Then there are numbers $u_{j k}, v_{j k}, w_{j k}, \lambda_{j k}$, $j=1, \ldots, \mu, k=1,2, \ldots$, with

and

$$
\begin{array}{cc}
\zeta_{k}^{0} \geqq \sum \lambda_{j k} u_{j k}\left(v_{j k}^{2}+w_{j k}^{2}\right), & -\zeta_{k}^{1}=\sum \lambda_{j k} u_{j k} v_{j k}, \\
-\zeta_{k}^{2}=\sum \lambda_{j k} u_{j k} w_{j k}, & \zeta_{k}^{3}=\sum \lambda_{j k} w_{j k}, \quad-\zeta_{k}^{4}=\sum \lambda_{j k} v_{j k}, \\
a \leqq u_{j k} \leqq b, \quad-\infty<v_{j k}, w_{j k}<+\infty, \quad \lambda_{j k} \geqq 0, & \sum \lambda_{j k}=1,
\end{array}
$$

$$
\zeta_{k}^{0} \rightarrow \zeta^{0}, \quad \zeta_{k}^{1} \rightarrow \zeta^{1}, \quad \zeta_{k}^{2} \rightarrow \zeta^{2}, \quad \zeta_{k}^{3} \rightarrow \zeta^{3}, \quad \zeta_{k}^{4} \rightarrow \zeta^{4} \quad \text { as } k \rightarrow \infty .
$$

If for $\infty$-many $k$ some $\lambda_{j k}=0$, then, by an exchange of indices we may as well assume that this occurs for the same $j$, say $j=1$, and by an extraction, we may as well assume that this occurs for all $k$, and in this case we are reduced to the same situation with $\mu$ replaced by $\mu-1$. Thus, it is not restrictive to assume 
that at most finitely many $\lambda_{j k}$ are zero, and finally, by abandoning a few terms of the sequence, we may as well assume $\lambda_{j k} \neq 0$ for all $j$ and $k$.

If there are $\infty$-many $\lambda_{j k}$ as small as we want, we can always assume that this occurs at least for $j=\mu$, and then we can extract a subsequence with $\lambda_{\mu k} \rightarrow 0$ as $k \rightarrow \infty$. If there are still $\infty$-many $\lambda_{j k}$, with $1 \leqq j \leqq \mu-1$, in the extracted subsequence with $\lambda_{j k}$ as small as we want, we can as well assume that this occurs for $j=\mu-1$, and by a new extraction we may assume $\lambda_{\mu-1, k} \rightarrow 0$ as $k \rightarrow \infty$. By repeating this process, we obtain a subsequence, say still $\left(\lambda_{j k}, j=1, \ldots, \mu\right), k=1,2, \ldots$, such that

$$
\begin{aligned}
1 \geqq \lambda_{j k} \geqq \gamma>0 & \text { for all } j=1,2, \ldots, \mu^{\prime}, \\
\lambda_{j k} \rightarrow 0 \text { as } k \rightarrow \infty & \text { for all } j=\mu^{\prime}+1, \ldots, \mu .
\end{aligned}
$$

Since $\sum \lambda_{j k}=1$ for every $k$, the first alternative must actually occur for at least one $j$, that is, $1 \leqq \mu^{\prime} \leqq \mu$. By a new extraction, we obtain now a subsequence, for which we use the same notation, such that

$$
\begin{aligned}
& \lambda_{j k} \rightarrow \lambda_{j} \geqq \gamma>0 \quad \text { as } k \rightarrow \infty, \quad j=1,2, \ldots, \mu^{\prime} . \\
& \lambda_{j k} \rightarrow 0 \text { as } k \rightarrow \infty, \quad j=\mu^{\prime}+1, \ldots, \mu .
\end{aligned}
$$

Since the numbers $u_{j k}$ are bounded, we can always extract the subsequence in such a way that we have also

$$
u_{j k} \rightarrow u_{j} \quad \text { as } \quad k \rightarrow \infty, \quad j=1, \ldots, \mu, a \leqq u_{j} \leqq b .
$$

Now $\zeta_{k}^{0} \rightarrow \zeta^{0}$, hence $\left[\zeta_{k}^{0}\right]$ is a bounded sequence, and

$$
\zeta_{k}^{0} \geqq \sum \lambda_{j k} u_{j k}\left(v_{j k}^{2}+w_{j k}^{2}\right) \text {. }
$$

We shall denote by $\sum^{\prime}, \sum^{\prime \prime}, \sum$ sums ranging over all $j=1, \ldots, \mu^{\prime}$, or $j=\mu^{\prime}+1, \ldots, \mu$, or $j=1, \ldots, \mu$, respectively. Hence (27) yields

$$
\zeta_{k}^{0} \geqq \sum^{\prime} \lambda_{j k} u_{j k}\left(v_{j k}^{2}+w_{j k}^{2}\right)
$$

with $\lambda_{j k} \rightarrow \lambda_{j} \geqq \gamma>0, u_{j k} \rightarrow u_{j} \geqq a>0, j=1, \ldots, \mu^{\prime}$, and [ $\left.\zeta_{k}^{0}\right]$ bounded. Hence, the $2 \mu^{\prime}$ sequences $\left[v_{j k}\right],\left[w_{j k}\right], j=1, \ldots, \mu^{\prime}$, are also bounded, and we can extract the subsequence in such a way that we have also

$$
v_{j k} \rightarrow v_{j}, \quad w_{j k} \rightarrow w_{j} \text { as } k \rightarrow \infty, \quad v_{j}, w_{j} \text { finite }, \quad j=1, \ldots, \mu^{\prime} .
$$

Actually, relation (27) yields also

$$
\zeta_{k}^{0} \geqq \sum^{\prime \prime} \lambda_{j k} u_{j k}\left(v_{j k}^{2}+w_{j k}^{2}\right),
$$

and hence the sequences

$$
\left[\lambda_{j k} u_{j k} v_{j k}^{2}\right], \quad\left[\lambda_{j k} u_{j k} w_{j k}^{2}\right], \quad j=\mu^{\prime}+1, \ldots, \mu,
$$

also are bounded, and so must be the sequences

$$
\left[\lambda_{j k} v_{j k}^{2}\right], \quad\left[\lambda_{j k} w_{j k}^{2}\right], \quad j=\mu^{\prime}+1, \ldots, \mu,
$$

since $0<a \leqq u_{j k} \leqq b$. Note that for any $j$, either $\left[v_{j k}\right]$ contains a subsequence which converges to zero, and in correspondence $\lambda_{j k} v_{j k} \rightarrow 0$, or $\left[v_{j k}\right]$ contains a subsequence bounded away from zero and in correspondence $\left[\lambda_{j k} v_{j k}\right]$ is also a 
bounded sequence. At least one of the two alternatives must occur. By repeating $2 \mu$ successive operations of extraction we can obtain, therefore, a final subsequence such that the sequences

$$
\left[\lambda_{j k} v_{j k}\right], \quad\left[\lambda_{j k} w_{j k}\right], \quad j=\mu^{\prime}+1, \ldots, \mu,
$$

are bounded. Then we can extract also a subsequence such that

$$
\hat{\lambda}_{j k} v_{j k} \rightarrow A_{j}, \quad \lambda_{j k} w_{j k} \rightarrow B_{j} \text { as } k \rightarrow \infty, \quad A_{j}, B_{j} \text { finite, } \quad j=\mu^{\prime}+1, \ldots, \mu .
$$

From relations (26) we deduce now as $k \rightarrow \infty$

$$
\begin{array}{rlrl}
\sum^{\prime \prime} A_{j} & =-\zeta^{4}-\sum^{\prime} \lambda_{j} v_{j}, & \sum^{\prime \prime} B_{j} & =\zeta^{3}-\sum^{\prime} \lambda_{j} w_{j}, \\
\sum^{\prime \prime} A_{j} u_{j} & =-\zeta^{1}-\sum^{\prime} \lambda_{j} u_{j} v_{j}, & \sum^{\prime \prime} B_{j} u_{j}=-\zeta^{2}-\sum^{\prime} \lambda_{j} u_{j} w_{j} .
\end{array}
$$

Let us prove that all numbers $A_{j}, B_{j}$ are zero. Assume this is not the case, and that at least one of the numbers $A_{j}, B_{j}, j=\mu^{\prime}+1, \ldots, \mu$, is $\neq 0$. Let us write the first relation (26) in the form

$$
\sum^{\prime \prime}\left[\left(\lambda_{j k} v_{j k}\right) u_{j k} v_{j k}+\left(\lambda_{j k} w_{j k}\right) u_{j k} w_{j k}\right] \leqq \zeta_{k}^{0}-\sum^{\prime} \lambda_{j k} u_{j k}\left(v_{j k}^{2}+w_{j k}^{2}\right),
$$

where the second member converges to

$$
\zeta^{0}-\sum^{\prime} \lambda_{j} u_{j}\left(v_{j}^{2}+w_{j}^{2}\right) .
$$

In the first member of (31) each of the products $\left(\lambda_{j k} v_{j k}\right) u_{j k} v_{j k},\left(\lambda_{j k} w_{j k}\right) u_{j k} w_{j k}$ is $\geqq 0$, hence (31) can be written in the form

$$
\sum^{\prime \prime}\left[\left|\lambda_{j k} v_{j k}\right| u_{j k}\left|v_{j k}\right|+\left|\lambda_{j k} w_{j k}\right| u_{j k}\left|w_{j k}\right|\right] \leqq m,
$$

where $m>0$ is some fixed number.

In (32), $\left|\lambda_{j k} v_{j k}\right| \rightarrow\left|A_{j}\right|,\left|\lambda_{j k} w_{j k}\right| \rightarrow\left|B_{j}\right|$, and $u_{j k} \rightarrow u_{j} \geqq a>0$, with $u_{j k} \geqq a>0$, $j=\mu^{\prime}+1, \ldots, \mu$, and all $k$, while $\lambda_{j k} \rightarrow 0$ as $k \rightarrow \infty, j=\mu^{\prime}+1, \ldots, \mu$. If one at least of the numbers $A_{j}, B_{j}$, say $A_{v}$, were different from zero, then $\left|v_{v k}\right| \rightarrow+\infty$ as $k \rightarrow \infty$, and

$$
\left|\lambda_{v k} v_{k}\right| \rightarrow\left|A_{v}\right| \neq 0, \quad u_{v k} \geqq a, \quad\left|v_{v k}\right| \rightarrow+\infty,
$$

would imply that the first number of (32) approaches $+\infty$ as $k \rightarrow \infty$, a contradiction. Thus $A_{j}=B_{j}=0, j=\mu^{\prime}+1, \ldots, \mu$, relations (30) become

and from (28) also

$$
\begin{aligned}
& -\sum^{\prime} \lambda_{j} u_{j} v_{j}=\zeta^{1}, \quad-\sum^{\prime} \lambda_{j} u_{j} w_{j}=\zeta^{2}, \\
& -\sum^{\prime} \lambda_{j} w_{j}=\zeta^{3}, \quad \sum^{\prime} \lambda_{j} v_{j}=\zeta^{4} \text {, }
\end{aligned}
$$

$$
\zeta^{0} \geqq \sum^{\prime} \lambda_{j} u_{j}\left(v_{j}^{2}+w_{j}^{2}\right), \sum^{\prime} \lambda_{j}=1 .
$$

In other words $\zeta=\left(\zeta^{0}, \ldots, \zeta^{4}\right)$ belongs to $\mathscr{Q}_{0}$ as a convex combination corresponding to some $\mu^{\prime}, 1 \leqq \mu^{\prime} \leqq \mu$ (in other words we can take $\lambda_{j}=0$ for $j=$ $\left.\mu^{\prime}+1, \ldots, \mu\right)$. We have proved that $Q_{0}$ is a closed set, and so are all sets $Q_{0}^{*}(x, y)$ as rigid displacements of $Q_{0}$.

Here the set $U^{\mu} \times \Gamma$ is a fixed closed set and hence certainly satisfies property $(U)$. Here the sets $\bar{Q}^{*}$ are rigid displacements of $Q_{0}$ by the vector

$$
\left(0,-W(y) B^{\prime}(x), 0,0,0\right) \text {. }
$$


Since $W(y) B^{\prime}(x)$ is a continuous function on cl $G$ we conclude that $\tilde{Q}^{*}$ certainly satisfies property $(Q)$.

By force of Theorem 3 with $\psi(x, y)=0$ and the corresponding remarks, we conclude that LURIE's problem has a weak optimal solution $\left(Z^{1}, Z^{2}\right)$ satisfying equations (13), satisfying the boundary conditions (18), minimizing the functional (14), with $\mu=6$, and such that

$$
Z_{x}^{1}, Z_{y}^{1}, Z_{x}^{2}, Z_{y}^{2} \in L_{2}(G) ; \quad Z^{1}, Z^{2} \in L_{2}\left(G_{N}\right) \text { for every } N
$$

and $Z^{1}, Z^{2} \in W_{2}^{1}\left(G_{N}\right)$ for every $N$.

Remark 11. The question as to whether the sets $\tilde{Q}^{*}$, or $\tilde{Q}^{*}$, are convex for some value of $\mu$ less than six has not been discussed above. Our existence statement holds for any $\mu \leqq 6$ for which the sets $\tilde{Q}^{*}$ or $\tilde{Q}^{*}$ are convex. The question as to whether weak solutions (that is, satisfying equations (13)) and corresponding values of the cost functional (14) can be approximated by means of solutions of equations (9) and corresponding values of the cost functional (12) will be answered elsewhere.

Research partially supported by AF-OSR grant $942-65$ at the University of Michigan. This paper was written during the author's stay at the "Institut Henri Poincaré", Paris, Spring 1967.

\section{References}

1. Cesari, L., Existence theorems for optimal solutions in Pontryagin and Lagrange problems. SIAM J. Control 3, 475-498 (1966).

2. CESARI, L., Existence theorems for weak and usual optimal solutions in Lagrange problems with unilateral constraints, I and II. Trans. Amer. Math. Soc. 124, 369-412, 413-429 (1966).

3. Cesari, L., Existence Theorems for Multidimensional Problems of Optimal Control. Differential Equations and Dynamical Systems (J. K. Hale and J. P. LA SAlle editors), 115-132. Academic Press 1967.

4. CeSARI, L., Existence theorems for multidimensional Lagrange problems. Journal of Optimization Theory and Applications 1, 87-112 (1967).

5. CeSARI, L., Sobolev spaces and multidimensional Lagrange problems of optimization. Annali Scuola Normale Sup. Pisa. (To appear.)

6. LURIE, K. A., The Mayer-Bolza problem for multiple integrals and the optimization of the performance of systems with distributed parameters. Prikl. Mat. Mek. 27, 842-853 (1963). English Translation, PMM, Pergamon Press 27, 1284-1299 (1963).

7. LURIE, K. A., Optimum control of conductivity of a fluid moving in a channel in a magnetic field. Prikl. Mat. Mek. 28, 258-267 (1964). English Translation, PMM, Pergamon Press 28, 316-327 (1964).

8. Lurie, K. A., The Mayer-Bolza Problem for Multiple Integrals: Some Optimum Problems for Elliptic Differential Equations Arising in Magnetohydrodynamics. Topics in Optimization, pp. 147-193 (edit. G. LerTmann). Academic Press 1967.

9. Rashevsky, P. K., Geometricheskaya Teoria Uravnenii Tchastnimi Proisvodnimi (Geometrical Theory of Partial Differential Equations). Gostekhizdat, Moscow 1947.

10. Thomas, T. Y., \& E. W. TitT, Systems of Partial Differential Equations and their Characteristic Surfaces. Ann. of Math. (2) 33, 1-80 (1932).

11. Cowling, T. G., Magnetohydrodynamics. New York: Wiley (Interscience) 1957.

Department of Mathematics University of Michigan Ann Arbor, Michigan 